\title{
SEPARATRIX AND LIMIT CYCLES OF QUADRATIC SYSTEMS AND DULAC'S THEOREM
}

BY

\author{
CARMEN CHICONE ${ }^{1}$ AND DOUGLAS S. SHAFER ${ }^{2}$
}

\begin{abstract}
Separatrix cycles for a planar quadratic vector field are studied. The results obtained are used to show that in any bounded region of the plane a quadratic vector field has at most a finite number of limit cycles.
\end{abstract}

1. Introduction. The second part of Hilbert's sixteenth problem [8] to find a bound for the number of limit cycles of the general polynomial vector field $X(x, y)=$ $P(x, y) \partial / \partial x+Q(x, y) \partial / \partial y$ in terms of the maximum degree of $P$ and $Q$, remains one of the most famous and most challenging problems in nonlinear differential equations. As a first step toward the solution of Hilbert's problem one would like to know that, given a specific pair $P$ and $Q, X$ has at most a finite number of limit cycles. If this is not the case there must be infinitely many limit cycles accumulating on either a periodic orbit, a critical point, or a separatrix cycle, or there must be a sequence of points, each on a distinct limit cycle, tending to infinity. In his 1881 Memoir [10] (which inspired Hilbert's problem) Poincaré introduced his loi de conséquence, now called the Poincaré return map, and used it to show that the first case cannot occur, i.e., limit cycles cannot accumulate on a periodic orbit. The argument is very simple. If $\rho$ denotes the return map on a local section $\Sigma$ transverse to the periodic orbit $\gamma$ of accumulation, then choosing a local coordinate $x$ on $\Sigma$ observe that $f(x)=\rho(x)-x$ is an analytic function whose zeros correspond to the periodic orbits of $X$ near $\gamma$. However, an analytic function which has an accumulation point of its zeros is identically zero, so the periodic orbits near $\gamma$ are not limit cycles.

Dulac in a lengthy article [6] claimed to have excluded the remaining cases. However, it is now widely recognized that his proofs are not complete. Thus, for an analytic vector field it is unknown if a critical point or a separatrix cycle can be the accumulation set of infinitely many limit cycles. We wish to thank Jorge Sotomayor for pointing out to us that Dulac's argument is correct (see \$4) in the special case when the analytic vector field has a separatrix loop at a hyperbolic saddle point.

Received by the editors December 15, 1981 and, in revised form, June 10, 1982.

1980 Mathematics Subject Classification. Primary 58F21; Secondary 34C05.

Key words and phrases. Limit cycle, quadratic system, separatrix cycle, Dulac's Theorem.

${ }^{1}$ Research supported in part by a grant from the Research Council of the Graduate School, University of Missouri.

${ }^{2}$ Research supported in part by a grant from the UNCC Foundation.

(C)1983 American Mathematical Society 0002-9947/82/0000-1275/\$08.25 
In this paper we study quadratic polynomial vector fields. For these quadratic systems it is impossible to have limit cycles accumulate at a critical point. In fact, if such a critical point exists the linear part of the vector field at the critical point vanishes identically or has pure imaginary eigenvalues. The first case is impossible because a homogeneous quadratic vector field has no limit cycles. The second case can be excluded for any analytic vector field [2, p. 254]. Thus, if a quadratic system has infinitely many limit cycles they accumulate on a separatrix cycle or they have an accumulation point at infinity.

Our work began by asking if a quadratic system can have even one limit cycle surrounded by a separatrix cycle (see the conjecture in $\$ 5$ ). This led us to examining the separatrix cycles themselves, with the, perhaps surprising, results stated in Theorems 3.1 and 3.2. These results exploit special properties of quadratic systems and they establish that infinitely many limit cycles may not accumulate on separatrix cycles. We note that the main arguments of Poincaré, Bendixson and Dulac were made for analytic systems. They used the fact that the vector field is a polynomial only to obtain an analytic extension to the Poincare sphere and to be certain that there are only finitely many critical points.

The first part of $\S 2$ lists special properties of quadratic systems. We also state and prove a new result on structure near a critical point, and list several immediate consequences for separatrix cycles. $\$ 3$ lists the two main results characterizing separatrix cycles containing more than one critical point, and $\$ 5$ concludes with examples and questions.

Our results offer some progress toward the topological classification of the possible phase portraits of a quadratic system in a region surrounded by a separatrix cycle. In particular, we obtain, as an application, the following partial solution of Dulac's problem.

TheOREM A. If a quadratic system has infinitely many limit cycles then the limit cycles have an accumulation point at infinity.

We remark in closing this section that in what follows, if $C$ is a simple closed curve we let int $C$ denote the (open) bounded region of the plane as divided by $C$.

Both authors wish to thank the referee for his very careful reading of the first version of this paper.

2. Quadratic systems. A quadratic system is one of the form

$$
\dot{x}=P(x, y), \quad \dot{y}=Q(x, y),
$$

where $P$ and $Q$ are relatively prime polynomials of degree at most two (and one of degree two). Equivalently we may consider the vector field $X(x, y)=P(x, y) \partial / \partial x$ $+Q(x, y) \partial / \partial y$ on $\mathbf{R}^{2}$. In any event we will refer to the system as $X$. We also let $\eta(t, p)$ denote the unique trajectory of $X$ starting at $p$, let $o_{+}(x)$ denote the positive semiorbit of $x, o_{-}(x)$ the negative semiorbit, and $o(x)$ the orbit of $x$.

We begin by stating, without proof, three fundamental results of the theory of quadratic systems; the proofs and appropriate references can be found in Coppel's survey [4].

The first result is elementary but is undoubtedly the most useful tool in the theory. 
TheOREM 2.1 (Tung's Lemma). (a) Three critical points of $X$ are never collinear.

(b) If l is a straight line which is not invariant, then the total number of critical points and contacts of $X$ on $l$ is at most two. If there are two such points then on the finite segment of $l$ cut off by them $X$ points into the opposite side of $l$ from that into which it points along either infinite segment of $l$.

THEOREM 2.2 (BERLINSKII''s THEOREM). If $X$ has the maximum number, four, of critical points, then they are all elementary (i.e., the Jacobian determinant at each one is nonzero). In particular, every saddle point is hyperbolic. Moreover, if the quadrilateral with vertices at the critical points is convex then two opposite vertices are saddles and the other two are antisaddles (nodes, foci or centers). But, if the quadrilateral is not convex then either the three exterior vertices are saddles and the interior vertex is an antisaddle or the exterior vertices are all antisaddles and the interior vertex is a saddle.

Theorem 2.3 (CENTER TheOREM). The system $X$ given by

$$
\begin{aligned}
& \dot{x}=y+b x^{2}+(2 c+\beta) x y+d y^{2}, \\
& \dot{y}=-\left[x+a x^{2}+(2 b+\alpha) x y+c y^{2}\right],
\end{aligned}
$$

has a center at the origin if and only if one of the following three conditions is satisfied:

(I) $a+c=b+d=0$,

(II) $\alpha(a+c)=\beta(b+d), a \alpha^{3}-(3 b+\alpha) \alpha^{2} \beta+(3 c+\beta) \alpha \beta^{2}-d \beta^{3}=0$,

(III) $\alpha+5(b+d)=\beta+5(a+c)=a c+b d+2\left(a^{2}+d^{2}\right)=0$.

Moreover if (I), (II) or (III) holds then $X$ has an integral (expressible in terms of elementary functions), hence does not have a limit cycle.

A special case of the following theorem is presented in Chicone-Tian [3]. We give here the general statement with a complete proof.

TheOREM 2.4 (Chicone-Shafer-TIAN). Let $p$ be a critical point of $X$.

(a) If the eigenvalues of the linear part $d X$ of $X$ at $p$ have nonzero imaginary part then $p$ is a focus or a center.

(b) If the eigenvalues of $d X$ at $p$ are real, then there is a null solution at $p$ (i.e., a trajectory tending to $p$ as $t \rightarrow+\infty$ or as $t \rightarrow-\infty$ ). For every null solution $\sigma$ at $p$ there is a line l such that either (i) $\sigma \subset l$, and $l$ is $X$-invariant along its entire length, or (ii) $\sigma$ is tangent to $l$ at $p, X$ has no critical points or contacts on $l$ besides $p$, and $X$ points to the same side of $l$ all along $l$. Moreover, in case (b) either there are one, two or three lines $l_{1}, l_{2}, l_{3}$ such that every null solution lies in or is tangent to one of them (the case of three lines occurs only if $X$ is homogeneous (has no linear part in coordinates such that $p=(0,0))$ and $l_{1}, l_{2}, l_{3}$ are invariant $)$, or there is a unique null solution in every direction at $p$, in which case $p$ is a hyperbolic source or sink and one, two or three invariant lines pass through $p$.

Proof. Part (a) is well known. Translating $p$ to $(0,0)$ and making a linear change of coordinates we may put $X$ into the form

$$
X(x, y)=\left(a x-b y+P_{2}(x, y)\right) \partial / \partial x+\left(b x+a y+Q_{2}(x, y)\right) \partial / \partial y
$$

where $b \neq 0$ and $P_{2}$ and $Q_{2}$ vanish together with their first partials at $(0,0)$. It is a straightforward exercise involving estimates on $\dot{\theta}$ and $\dot{r}$ near $(0,0)$ to show that 
trajectories of $X$ near $(0,0)$ cross every ray from $(0,0)$ infinitely often. As noted in the introduction, a rotation point of a quadratic system must be a center, so part (a) is established.

Hence, suppose that the eigenvalues of $d X$ at $p$ are real. Translating $p$ to $(0,0)$ we may assume $X$ has the form

$$
\begin{aligned}
X(x, y)= & \left(a x+b y+e x^{2}+f x y+k y^{2}\right) \partial / \partial x \\
& +\left(c x+d y+m x^{2}+n x y+p y^{2}\right) \partial / \partial y,
\end{aligned}
$$

hence

$$
d X(0,0)=\left(\begin{array}{ll}
a & b \\
c & d
\end{array}\right) .
$$

The tangential component $\dot{\theta}$ of $X$ at the point with coordinates $(x, y) \neq(0,0)$ is

$$
\begin{aligned}
\dot{\theta}(x, y) & =[y P(x, y)-x Q(x, y)] \cdot\left(x^{2}+y^{2}\right)^{-1} \\
& =\left[c x^{2}+(d-a) x y-b y^{2}+h(x, y)\right] \cdot\left(x^{2}+y^{2}\right)^{-1} \\
& =[g(x, y)+h(x, y)] \cdot\left(x^{2}+y^{2}\right)^{-1}
\end{aligned}
$$

where $h(x, y)$ is a homogeneous polynomial of degree three in $x$ and $y$.

The discriminant of $g$ is $(d-a)^{2}+4 b c$, which is precisely the discriminant of $d X(0,0)$, hence by hypothesis is nonnegative. Thus $g(x, y)$ has at least one line through $(0,0)$ along which it vanishes. Since $h(x, y)$ is a homogeneous cubic, it too vanishes along a line through $(0,0)$.

If $g$ and $h$ vanish together along a line $l$ through $(0,0)$, then $l$ is invariant for $X$ and each portion near $(0,0)$ is a null solution at $(0,0)$. If $h$ is nonzero on every radial line $l$ along which $g$ vanishes, then $X$ has no critical points or contacts on $l$, and since $h$ is a cubic $X$ points to the same side of $l$ all along $l$. Moreover, there exist distinct lines $l_{1}$ and $l_{2}$ such that $g$ vanishes along $l_{1}, h$ vanishes along $l_{2}$, and $g \cdot h \neq 0$ in two of the sectors $\alpha$ and $\alpha^{\prime}$, opposite one another, which are the interiors of the angles formed by $l_{1}$ and $l_{2}$. In fact (since $g \cdot h$ has odd degree) $g \cdot h<0$ in one sector, say $\alpha$, and $g \cdot h>0$ in the other. Let $\rho_{1}$ and $\rho_{2}$ denote the half-lines bordering on $\alpha$. Then $\dot{\theta}$ has opposite sign along $\rho_{1}$ and $\rho_{2}$, hence $\alpha$ is positively or negatively invariant, say positively (replacing $X$ by $-X$ if necessary).

Let $C$ be any circle about $(0,0)$ isolating it from other critical points. If there is no point on $\rho_{1}$ or $\rho_{2}$ in int $C$ whose positive semiorbit tends to $(0,0)$, the PoincaréBendixson Theorem implies that the positive semiorbit of every such point must permanently leave $\alpha \cap[C \cup$ int $C]$. Then choose any sequence $\left\{x_{j}\right\}_{j=1}^{\infty} \subset \rho_{1}$ tending monotonically to $(0,0)$ and for each $j$ let $x_{j}^{\prime}$ be the last intersection of the positive semiorbit through $x_{j}$ with $C$. There is an accumulation point $x \in \alpha \cap C$ of the sequence $\left\{x_{j}^{\prime}\right\}_{j=1}^{\infty}$, and an argument as in Hartman [7, p. 167] shows that the negative semiorbit through $x$ exists on $(-\infty, 0]$ and tends to $(0,0)$ as $t \rightarrow-\infty$. This establishes the existence of a null solution.

If $\sigma$ is a null solution at $(0,0)$, it cannot spiral about $(0,0)$ because of the behavior of $X$ along the line (or lines) where $g$ vanishes, as described above. Neither can $\sigma$ oscillate back and forth across two distinct radial rays repeatedly, since to do so 
would require more than two contacts of $X$ on each ray, which is impossible by Tung's Lemma (2.1). Thus $\sigma$ has a tangent at $(0,0)$.

But now if $l$ : $\theta=\theta_{0}$ is the tangent line for a null solution $\sigma$, then clearly $g$, the lowest order terms of $\dot{\theta}$, is zero along $l$. For this a straightforward estimate involving $\dot{\theta}$ and $\dot{r}=r k(r, \theta), k$ smooth, shows that if $g\left(\cos \theta_{0}, \sin \theta_{0}\right)>0$, then there exists $\varepsilon>0$ such that the positive semiorbit through any point on the line $l_{-}: \theta=\theta_{0}-\varepsilon$, sufficiently close to $(0,0)$, crosses the line $l_{+}: \theta=\theta_{0}+\varepsilon$ with monotonically increasing $\theta$ coordinate, hence there is no null solution tangent to $l$. But now if $g$ vanishes along $l$, then the behavior of $X$ along $l$ must be as claimed.

If $g \neq 0$ the last assertion of the theorem is immediate. If $g \equiv 0$, then when $a \neq 0$ the last assertion is [1, Theorem 64, p. 331], and when $a=0$ then $X$ is homogeneous, $h \neq 0$ (else $X$ is scaled linear) plays the role of $g$, and the result follows similarly. Q.E.D.

The following useful lemma describes the restrictions that existence of an elliptic sector at a critical point places on a nonhomogeneous quadratic system.

LEMMA 2.5. If $X$ is nonhomogeneous and has an elliptic sector at an isolated critical point $p$, then there is exactly one hyperbolic sector and exactly one elliptic sector at $p$, every null solution at $p$ is tangent to a line $l$ which is $X$-invariant and contains no critical points besides $p$, and $X$ has at most one critical point besides $p$.

Proof. This result follows primarily from the classification theorems of Andronov et al. [1, Chapter IX]. We may translate $p$ to $(0,0)$, where $X$ must have a nonzero linear part with trace and determinant zero. Thus by a linear change of coordinates, if necessary, $X$ has the form

$$
X(x, y)=\left[y+a x^{2}+b x y+c y^{2}\right] \partial / \partial x+\left[d x^{2}+e x y+f y^{2}\right] \partial / \partial y,
$$

so that

$$
\begin{aligned}
\left(x^{2}+y^{2}\right) \cdot \dot{\theta}(x, y) & =g(x, y)+h(x, y) \\
& =-y^{2}+\left[d x^{3}+(e-a) x^{2} y+(f-b) x y^{2}-c y^{3}\right] ;
\end{aligned}
$$

$g(x, y)$ vanishes only along the line $l: y=0$, so by the proof of Theorem 2.4 every null solution is tangent to $l$.

$X$ is vertical along the curve $y=\phi(x)=0+0 \cdot x-a x^{2}+\cdots$; the nonzero coefficient of $\partial / \partial y$ along this curve is therefore $\psi(x)=d x^{2}+\cdots$. By Theorems 66 and 67 of Andronov et al. [1,pp. 357, 362], $d=0$. But then $\dot{\theta}$ vanishes along $l$, along which $X$ is now $X(x, 0)=a x^{2} \partial / \partial x$, vanishing only for $x=0$.

Since $(e, f)=(0,0)$ is impossible $X$ has precisely one or two critical points. Q.E.D.

The intuitive notion of a separatrix cycle is clear; a separatrix is a trajectory which forms part of the boundary of a hyperbolic sector at some critical point, and a separatrix cycle is a simple closed curve consisting of separatrices and critical points, which is traversed by the flow in a definite direction. While any separatrix cycle surrounding a limit cycle must also surround a critical point, a general separatrix cycle need not do so. For example, the interior of the separatrix cycle could be filled 
with paths which form an elliptic sector at one critical point on the cycle. It is clear that infinitely many limit cycles can accumulate on a separatrix cycle on a given side only if the Poincare return map is defined on that side for a transverse section at any noncritical point of the cycle. (If a separatrix cycle $S_{1}$ surrounds and partly includes a separatrix cycle $S_{2}$, so that every point of $S_{2}$ is an accumulation point for the set of points on the limit cycles, but only some of the points of $S_{1}$ are (e.g., part of $S_{1}$ bounds parabolic sectors at two points of $S_{2}$, so $\left.\operatorname{int}\left(S_{2}\right) \varsubsetneqq \operatorname{int}\left(S_{1}\right)\right)$, then we say that the limit cycles accumulate on $S_{2}$ but not on $S_{1}$.) Therefore we make the following definition.

Definition 2.6. Let $S$ be a separatrix cycle, dividing the plane into $R_{1}$ and $R_{2}$. Then $S$ is a continuable separatrix cycle if for every $p \in S$ there is an arc without contact $\Sigma_{p}$ and a subarc $\tilde{\Sigma}_{p} \subset \Sigma_{p}$ containing $p$ such that the Poincare return map is defined on $\tilde{\Sigma}_{p} \cap R_{1}$ into $\Sigma_{p}$ (or similarly for $R_{2}$ and every point $p$ in $S$ ).

THEOREM 2.7. Any separatrix cycle of a quadratic vector field $X$ contains either one, two or three critical points.

Proof. A quadratic system has at most four critical points. If a separatrix cycle contains four critical points Berlinskiî's Theorem implies that at least one is a node, focus or center, a contradiction. Q.E.D.

THEOREM 2.8. If $S$ is a continuable separatrix cycle of a quadratic vector field $X$ and $p, q$ are two critical points on $S$, then the line segment $L$ joining $p$ and $q$ is a portion of $S$.

Proof. Tung's Lemma implies that int $S$ is a convex region. Hence, if the line segment $L$ is not contained in $S$, then $L-\{p, q\} \subseteq$ int $S$. Since $S$ is continuable a return map will be defined on open segments of $L$ near $p$ and near $q$. But, this implies that $X$ crosses $L$ with opposite orientations on these segments contrary to Tung's Lemma. Q.E.D.

LEMMA 2.9. Limit cycles accumulating on a separatrix cycle $S$ of a quadratic system do so from int $S$.

PROOF. This follows immediately from Theorem 2.4. Q.E.D.

We note in closing this section that major results for periodic orbits of quadratic systems $[3,4]$ are true, as well, for path-polygons (simple closed curves composed entirely of trajectories, including critical points). Notably, if $C$ is a path-polygon then int $C$ is a convex region and contains at most one critical point, which must be a focus or a center (this follows from 2.4).

We also remark that every critical point of a quadratic system is isolated; for the existence of infinitely many critical points implies that $P$ and $Q$ have a common linear factor, hence $X$ is a scaled linear system.

3. Separatrix and limit cycles. In this section we characterize separatrix cycles which contain two or three critical points, and establish Dulac's Theorem in these cases. 
THEOREM 3.1. Let $S$ be a separatrix cycle containing three critical points.

Then $S$ is a triangle with hyperbolic critical points at the vertices, there is a critical point in int $S$, which is a center, and every other trajectory in int $S$ is periodic.

Proof. Let $S$ contain critical points $p, q$ and $r$, and suppose the orientation of $S$ induced by the flow is from $p$ to $q$ to $r$ (i.e., $p$ is the $\alpha$-limit set and $q$ is the $\omega$-limit set of some point in $S$, and so on). We establish the existence of a critical point in int $S$ by contradiction.

Hence, suppose $S$ surrounds no critical point. Then it surrounds no closed orbit, so by the Poincare-Bendixson Theorem any point in int $S$ tends to a critical point in both forward and reverse time. Fix $w \in$ int $S \cap$ int $\triangle p q r$ and for definiteness say $\eta(t, w) \rightarrow p$ as $t \rightarrow+\infty$.

Case I. $\eta(t, w) \rightarrow p$ as $t \rightarrow-\infty$. Then $X$ has an elliptic sector at $p$, which is impossible by Lemma 2.5 .

Case II. $\eta(t, w) \rightarrow q$ as $t \rightarrow-\infty$. By convexity of int $S$ the portion of $S$ from $p$ to $q$ either lies on the opposite side of the line $l$ through $p$ and $q$ from $r$, or is the line segment (including endpoints) $L$ joining $p$ and $q$.

Let $S^{\prime}$ be the path-polygon formed by $q$, the orbit $o(w)$ of $w, p$, and the portion of $S$ from $p$ to $q$. Since $\operatorname{int}\left(S^{\prime}\right)$ is convex, $o(w)$ must lie on the same side of $l$ as $r$.

If $S^{\prime} \cap L=\{p, q\}$ then $L$ divides $\operatorname{int}\left(S^{\prime}\right)$ into two open regions, $A$ : that bounded by $L$ and $o(w)$, and $B$ : that bounded by $L$ and the portion of $S$ from $p$ to $q$. If $L \subset S, A \neq \varnothing, B=\varnothing$ and the flow is from $p$ to $q$ along $L$.

The convexity of regions bounded by path-polygons shows $S^{\prime} \cap L=\{p, q\}$ or $S^{\prime} \cap L$ (i.e. $L \subset S$ ). This together with convexity of regions bounded by pathpolygons and the impossibility of behavior as in Case I implies that for $y \in A$, $\eta(t, y) \rightarrow p$ as $t \rightarrow+\infty$ and $\eta(t, y) \rightarrow q$ as $t \rightarrow-\infty$, while for $y \in B, \eta(t, y) \rightarrow q$ as $t \rightarrow+\infty$ and $\eta(t, y) \rightarrow p$ as $t \rightarrow-\infty$. Moreover, $L$ contains no critical point or contact except $p$ and $q$ (Lemma 2.1).

Suppose $B=\varnothing$ so that the flow on $L$ is from $p$ to $q$. Let $z \in L-\{p, q\}$ and choose a small arc without contact $\Sigma$ at $z$ and a point $v \in \Sigma \cap A$ near $z$. Then $q$ is outside the simple closed curve $C$ formed by $z$, the portion of $\Sigma$ from $z$ to $v, v, o_{+}(v)$, $p$, and the segment of $L$ from $p$ to $z$, whereas for $t<0, \eta(t, v)$ is inside $C$, contradicting the fact that $\eta(t, v) \rightarrow q$ as $t \rightarrow-\infty$.

When $B$ is not empty, the direction of flow along $L$ is not determined, but either choice yields a contradiction. Thus Case II is impossible.

Case III. $\eta(t, w) \rightarrow r$ as $t \rightarrow-\infty$. By convexity of interiors of path-polygons either $w$ is in the line through $p$ and $r$ or is on the opposite side of this line from $q$, contradicting the choice of $w$. Thus $S$ surrounds a unique critical point which by 2.4 must be a focus or center.

By Berlinskiü's Theorem (2.2), $p, q$ and $r$ are elementary critical points, hence are hyperbolic saddles. It follows easily that $S$ is continuable, hence by Theorem 2.8 is a triangle with hyperbolic saddles at the vertices.

By a linear change of coordinates the vertices of the triangle can be placed at $(0,0),(0,1)$ and $(1,0)$. Then the fourth critical point has coordinates $(\alpha, \beta)$, some $\alpha$ 
and $\beta$ satisfying $0<\alpha, \beta, \alpha+\beta<1$. Invariance of the coordinate axes and the line with equation $x+y=1$ implies that $X$ has the form

$$
X(x, y)=a x\left(x+\frac{1-\alpha}{\beta} y-1\right) \frac{\partial}{\partial x}+b y\left(\frac{1-\beta}{\alpha} x+y-1\right) \frac{\partial}{\partial y}
$$

for some $a$ and $b$ satisfying $a \alpha+b \beta=0$.

Scaling by $\frac{1}{b}$ and translating $(\alpha, \beta)$ to the origin gives $X$ the form

$$
\begin{aligned}
X(x, y)= & {\left[-\beta x+(\alpha-1) y-\frac{\beta}{\alpha} x^{2}-\left(\frac{1-\alpha}{\alpha}\right) x y\right] \frac{\partial}{\partial x} } \\
& +\left[\frac{\beta}{\alpha}(1-\beta) x+\beta y+\left(\frac{1-\beta}{\alpha}\right) x y+y^{2}\right] \frac{\partial}{\partial y} .
\end{aligned}
$$

Applying the linear change of coordinates $\bar{x}=x, \bar{y}=D^{-1}[\beta x+(1-\alpha) y]$, where $D=\alpha^{-1 / 2}[\beta(1-\alpha-\beta)]^{1 / 2}$, in the new coordinates (dropping the overbars) $X$ has the form

$$
X(x, y)=\left[-D y-\frac{D}{\alpha} x y\right] \frac{\partial}{\partial x}+\left[D x+\frac{D}{\alpha-1} x^{2}+\frac{\alpha+2 \beta-1}{\alpha(\alpha-1)} x y-\frac{D}{\alpha-1} y^{2}\right] \frac{\partial}{\partial y} .
$$

By the Center Theorem (2.3), part (I) there is a center at the critical point inside the triangle.

An integral for the system (whose existence is stated in the Center Theorem (2.3)) is $I(x, y)=x^{r} y^{s}(1-x-y)$, where $r=\alpha(1-\alpha-\beta)^{-1}$ and $s=\beta(1-\alpha-\beta)^{-1}$, hence $X$ has no limit cycles and the periodic orbits surrounding the center fill up int $S$. Q.E.D.

THEOREM 3.2. Let $S$ be a separatrix cycle containing exactly two critical points $p$ and $q$.

(a) If there is no critical point in int $S$ then $X$ has an invariant line through $p$; at $p$ there is exactly one hyperbolic and one elliptic sector; $q$ is a hyperbolic saddle; and every trajectory in int $S$ tends to $p$ in forward and reverse time.

(b) If there is a critical point $v$ in int $S$, then either (i) one of $p$ and $q$ is a hyperbolic saddle point, the other is a saddle node, $v$ is a focus and $S$ surrounds at most finitely many closed orbits; or (ii) both $p$ and $q$ are hyperbolic saddle points and $S$ is continuable with $S$ surrounding infinitely many closed orbits only if $v$ is a center and every other trajectory in int $S$ is periodic.

Proof of 3.2. Suppose int $S$ contains no critical point of $X$, and fix $w \in$ int $S$. By the Poincaré-Bendixson Theorem $\eta(t, w)$ tends to either $p$ or $q$ as $t$ tends to either plus or minus infinity. For definiteness say $\eta(t, w) \rightarrow p$ as $t \rightarrow+\infty$.

Case I. $\eta(t, w) \rightarrow p$ as $t \rightarrow-\infty$. Then there is an elliptic sector at $p$, which we may assume is $(0,0)$. By the proof of Lemma $2.5, X$ may be written (after a linear change of coordinates, if necessary)

$$
X(x, y)=\left(y+a x^{2}+b x y+c y^{2}\right) \partial / \partial x+\left(e x y+f y^{2}\right) \partial / \partial y
$$

where $a \neq 0$. The $x$-axis is invariant.

The curve along which $X$ is vertical is

$$
y=\phi(x)=0+0 \cdot x-a x^{2}+a b x^{3}+\cdots .
$$


The vertical component of $X$ along $y=\phi(x)$ is

$$
\psi(x)=-a e x^{3}+\left(a b e+a^{2} f\right) x^{4}+\cdots,
$$

and the divergence of $X$ along $y=\phi(x)$ is

$$
\sigma(x)=(2 a+e) x+\cdots .
$$

Applying Theorem 66 of Andronov et al. [1, p. 357] we must have $a e>0$. Then $q$ is located at $\left(x_{0}, y_{0}\right)=\left(e f \alpha,-e^{2} \alpha\right)$, where $\alpha=\left(a f^{2}-b e f+c e^{2}\right)^{-1}$, and $\operatorname{det} d X(q)=$ $-e^{3} \alpha=e \cdot y_{0}<0$, hence $q$ is a hyperbolic saddle. But then, for any point $z \in$ int $S$, $\eta(t, z)$ does not tend to $q$ as $t$ tends to either plus or minus infinity, hence by the Poincaré-Bendixson Theorem tends to $p$ in each direction.

Case II. $\eta(t, w) \rightarrow q$ as $t \rightarrow-\infty$. Let $L$ be the line segment (including endpoints) from $p$ to $q$. If $S \cap L=\{p, q\}$ then $L$ divides int $S$ into two regions, $A$ : that bounded by $L$ and the portion of $S$ oriented by the flow from $q$ to $p$, and $B$ : that bounded by $L$ and the portion of $S$ oriented by the flow from $p$ to $q$. If $S \cap L=L$, one of $A$ and $B$ is empty and the other nonempty. By the convexity of regions bounded by path-polygons $w \notin B$, and either (a) $o(w)=L-\{p, q\}$ or (b) $o(w) \cap$ $L=\varnothing$. There is no elliptic sector at either $p$ or $q$, in case (a) because the line through $p$ and $q$ is invariant (violating Lemma 2.5), in case (b) because $w \in$ int $S$ but $\eta(t, w) \rightarrow p$ as $t \rightarrow+\infty$ and $\eta(t, w) \rightarrow q$ as $t \rightarrow-\infty$ (violating the last statement in Case I). But now the same sort of reasoning as in Case II of the proof of Theorem 3.1 yields a contradiction.

Thus only Case I occurs, establishing the statement of the theorem in the event int $S$ contains no critical point of $X$.

Now suppose there is a critical point $v \in$ int $S$. If $S$ is not continuable then using part (a) of this theorem just established clearly there is a parabolic sector at either $p$ or $q$ having nonempty intersection with int $S$. Say the parabolic sector occurs at $p$, and assume that $p=(0,0)$. Then $d X(0,0)$ is nonzero but has determinant zero. It follows that $S$ is not the accumulation set of infinitely many periodic orbits and that $p$ is a saddle node with $\operatorname{tr} d X(0,0) \neq 0$. (If $\operatorname{tr} d X(0,0)=0$ use [1, Theorems 66,67, pp. 357, 362] to see that $X$ has, after a linear change of coordinates, the form

$$
\left[y+a x^{2}+b x y+c y^{2}\right] \partial / \partial x+\left[e x y+f y^{2}\right] \partial / \partial y .
$$

Thus, $X$ has at most two critical points contrary to the assumption. If $\operatorname{tr} d X(0,0) \neq 0$ use [1, Theorem 65, p. 340] to conclude that $p$ is a saddle node.) Since $v$ is surrounded by $S, 2.4$ implies $v$ is either a focus or a center. However, if $v$ is a center, the Center Theorem implies the existence of a first integral for $X$ and, hence, $p$ could not be a saddle node. Thus, $v$ is a focus. In this situation $q$ must be a hyperbolic saddle point. In fact, if $X$ is a quadratic system with three critical points then $X$ has at most one nonelementary critical point. This follows from the fact that the conics $P(x, y)=0, Q(x, y)=0$ where $X=(P, Q)$ intersect with multiplicity two at a nonelementary critical point. Since $v$ is a focus and $S$ is not continuable, $S$ surrounds at most finitely many closed orbits. This establishes (b)(i) when $S$ is not continuable.

If $S$ is continuable, by Theorem 2.8 it contains the line segment joining $p$ and $q$. Since $X$ is a polynomial vector field this implies that the line $l$ through $p$ and $q$ is 
invariant. By an affine change of coordinates we may place $v$ at the origin and make $l$ the line with equation $1+x=0$, so that

$$
X(x, y)=(x+1)(r x+s y) \partial / \partial x+Q(x, y) \partial / \partial y
$$

where $s \neq 0$ (else the $y$-axis is invariant, contrary to the existence and position of $S$ ). By the linear change of coordinates that fixes $x$ and replaces $y$ by $-r x / s+y / s$ we place $X$ in the form

$$
X(x, y)=(x+1) y \frac{\partial}{\partial x}+\left(m x+n y+a x^{2}+b x y+c y^{2}\right) \frac{\partial}{\partial y}
$$

where $c \neq 0$ (else there would be only one critical point on $l$ ). The linear part of $X$ at any point is

$$
d X(x, y)=\left(\begin{array}{cc}
y & x+1 \\
m+2 a x+b y & n+b x+2 c y
\end{array}\right) .
$$

Since $X$ has a focus or a center at $(0,0)$, by $(1)$,

$$
\operatorname{det}\left(\begin{array}{cc}
0 & 1 \\
m & n
\end{array}\right)=-m>0
$$

(since $(m, n)=(0,0)$ is clearly impossible). Also, at the critical points $p$ and $q$ on $l$,

$$
d X(-1, y)=\left(\begin{array}{cc}
y & 0 \\
* & n-b+2 c y
\end{array}\right)
$$

so the eigenvalues are $y$ and $n-b+2 c y$, where the ordinates of the critical points are given by

$$
y=\frac{(b-n) \pm \sqrt{(b-n)^{2}-4 c(a-m)}}{2 c}=\frac{B \pm \sqrt{D}}{2 c} .
$$

The characteristic exponent of any closed orbit $\gamma:(x(t), y(t))$ in int $S$ is the number

$$
\begin{aligned}
h_{\gamma} & =\frac{1}{T} \int_{0}^{T} \operatorname{div} X(x(t), y(t)) d t \\
& =n+\frac{b}{T} \int_{0}^{T} x(t) d t+\frac{1+2 c}{T} \int_{0}^{T} y(t) d t .
\end{aligned}
$$

Since

$$
\int_{0}^{T} y(t) d t=\int_{0}^{T} \frac{\dot{x}(t)}{1+x(t)} d t=0
$$

it follows that

$$
h_{\gamma}=n+\frac{b}{T} \int_{0}^{T} x(t) d t .
$$

Since the critical points at $p$ and $q$ are not nodes, foci, or centers either one can fail to be hyperbolic only if the linear part of $X$ has a zero eigenvalue there. But $n-b+2 c y=0$ is impossible since by (2) it implies that $D=0$, contrary to the existence of two distinct critical points on $l$. Thus there is nonhyperbolicity at $p$ or $q$ 
if and only if $y=0$ in (2), which occurs if and only if $a=m$ and $b \neq n$ (else $D=0$ ). In such a case, after changing coordinates and rescaling, we find, by Theorem 65 of Andronov et al. [1, p. 340], that $m(b-n) \neq 0$ implies that the critical point on $S$ at $(-1,0)$ is a saddlenode whose parabolic sector has empty intersection with int $S$ (as it must, if int $S$ is to be convex); the eigenvalues of the linear part of $X$ at the remaining critical point of $S$ are the nonzero numbers $b-n$ and $(b-n) / c$, so it is a hyperbolic saddle point. When $a \neq m$ of course $y \neq 0$ in (2), so both $p$ and $q$ are hyperbolic saddle points.

First consider the case that one of $p$ and $q$ is a saddle node, so that $a=m$ and $b \neq n$. If $b=0$ then by (3) every closed orbit in int $S$ has characteristic exponent $n \neq 0$. But $n=\operatorname{tr} d X(0,0)$, hence every closed orbit in int $S$ has the same stability as the origin. Since this is impossible, int $S$ contains no closed orbit when $b=0$. If $b \neq 0$, set $\varepsilon=\min (|(n-b) / 2 b|, 3)>0, K=\sup \{x \mid(x, y) \in S\}$, and let $l^{\prime}$ denote the line with equation $x=-1+\varepsilon / 4$. By convexity of int $S$ and the choice $\varepsilon \leqslant 3, S \cap l^{\prime}$ consists of two points $u$ and $w$, the former with positive ordinate, the latter with negative ordinate; $w=\eta(\tau, u)$ for some unique $\tau>0$. Since the parabolic sector at the saddle node lies outside $S$, any closed orbit $\gamma:(x(t), y(t))$ in int $S$ sufficiently close to $S$ must intersect $l^{\prime}$ exactly twice, once near $u$ (leaving the strip $\{(x, y) \mid-1<x<-1+\varepsilon / 4\})$ and once near $w$ (entering the same strip). If $T$ is the least period of $\gamma$ and $T_{1}$ is the time of traversal of $\gamma$ from the point of crossing of $l^{\prime}$ near $u$ to the point of crossing near $w$, then for $\gamma$ sufficiently close to $S,\left|T_{1}-\tau\right|<1$ and $T$ is so large that

$$
\left|\frac{1}{T} \int_{0}^{T_{1}} x(t) d t\right|<\frac{\tau+1}{T} k<\frac{\varepsilon}{2} .
$$

On the other hand, choosing the motion on $\gamma$ so that $(x(0), y(0)) \in l^{\prime}$ near $u$, $-1<x(t)<-1+\varepsilon / 4$ when $T_{1}<t<T$, so that for $\gamma$ close enough to $S, T$ is so large that

$$
-1<\frac{1}{T} \int_{T_{1}}^{T} x(t) d t<-1+\frac{\varepsilon}{2} .
$$

Combining the two inequalities,

$$
-1-\varepsilon<\frac{1}{T} \int_{0}^{T} x(t) d t<-1+\varepsilon
$$

so that, by our choice of $\varepsilon$ and equation (3)

$$
\left|(n-b)-h_{\gamma}\right|<|(n-b) / 2|,
$$

hence all closed orbits in int $S$ sufficiently close to $S$ have nonzero characteristic exponent of the same sign as $n-b \neq 0$, and it is impossible for infinitely many closed orbits to accumlate on $S$. But by hyperbolicity of one of $p$ and $q$ and the nonintersection of the parabolic sector at the other with int $S$, there is no other separatrix cycle $S^{\prime}$ in int $S$ on which limit cycles can accumlate, so there are finitely many limit cycles in int $S$. Again, presence of the saddle node implies $v$ is not a center, so $S$ surrounds finitely many closed orbits. Hence, finally, let us prove that $S$ 
surrounds finitely many limit cycles in the remaining case that $a \neq m$ and $p$ and $q$ are hyperbolic saddle points.

As before, if $S$ surrounds infinitely many limit cycles they must accumulate on $S$, which is then neither stable nor unstable on the inside. But it is well known (Dulac [6], Reyn [11]) that such semistability implies that the product of the positive eigenvalues of $d X$ at $p$ and $q$ equals the product of the negative eigenvalues there. By (2) this implies $b=n$. Then by (3), when $n \neq 0$ the characteristic exponent of every closed orbit in int $S$ is nonzero and has the sign of $n$; since $n=\operatorname{tr} d X(0,0)$, again there can be only finitely many limit cycles when $n \neq 0$. But if $n=0$, then $b=n=0$ and $X$ is

$$
X(x, y)=(x+1) y \partial / \partial x+\left(m x+m x^{2}+c y^{2}\right) \partial / \partial y .
$$

Integral curves are symmetric (as point-sets) with respect to the $x$-axis, so $X$ has a center at $(0,0)$ and no limit cycles (or apply the Center Theorem (2.3)). Thus $S$ surrounds only finitely many limit cycles when $p$ and $q$ are hyperbolic saddle points.

Finally, if $S$ surrounds infinitely many closed orbits (but only finitely many limit cycles), then these closed orbits have accumulation sets consisting of isolated critical points, periodic orbits, or separatrix cycles. Since there are only finitely many critical points and separatrix cycles at least one closed orbit $\gamma$ is an accumulation set for closed orbits, and analyticity of the flow implies that $\gamma$ lies in a maximal open annulus $A$ consisting of closed orbits. The outer boundary $\partial A^{+}$is an invariant set, hence is either a closed orbit or a separatrix cycles $S^{\prime}$. By analyticity of the flow $\partial A^{+}$ is not a closed orbit; hence $\partial A^{+}=S^{\prime}$, and by reasoning previously given, in fact, $S^{\prime}=S$. The inner boundary $\partial A^{-}$of $A$ similarly cannot be a closed orbit, and by Lemma 2.9 is not a separatrix cycle. Thus $\partial A^{-}=v$, which is thus a center, and every other trajectory in int $S$ is periodic. Q.E.D.

COROLlaRY 3.3. If $S$ is a separatrix cycle containing exactly two critical points, then there are at most finitely many limit cycles in int $S$.

4. The separatrix loop. We consider in this section the case where the separatrix cycle $S$ contains a single critical point. Our goal is to show that, for a quadratic system $X, S$ is not the accumulation set of infinitely many limit cycles. The next theorem is analogous to the theorem of Reyn [11] on the stability of separatrix cycles with hyperbolic critical points and it is a consequence of the ideas developed by Andronov [2, \$29]. This seems to be well known but not easily found in the literature.

THEOREM 4.1. If $S$ is a separatrix loop at a critical point $p$ (possibly nonhyperbolic) of a smooth planar vector field $X$ and if $\operatorname{div} X(p) \neq 0$, then $S$ is not the accumulation set of infinitely many periodic orbits.

Proof. Assume $S$ is the accumulation set of infinitely many periodic orbits and note that $S$ must be continuable. Since $\operatorname{div} X(p) \neq 0$, by considering $-X$ if necessary, assume $\operatorname{div} X(p)=\sigma>0$. Choose a disk $D$ at $p$ such that $w \in D$ implies $\operatorname{div} X(w)>\sigma / 2$. Choose two local orthogonal trajectories of $X, l_{0}$ on the local unstable separatrix forming the loop and $l_{1}$ on the local stable separatrix. Let $p_{0}$ and 
$p_{1}$ denote the respective intersections of $l_{0}$ and $l_{1}$ with $S$, assume $p_{0}$ and $p_{1} \in D$ and that integral curves starting on $l_{1}$ near $p_{1}$ stay in $D$ until they meet $l_{0} ; \eta\left(\tau, p_{0}\right)=p_{1}$ for some unique $\tau>0$. Define a coordinate $u$ on $l_{0}$ so that $p_{0}$ has coordinate 0 and such that a positive coordinate corresponds to a point on the inside of the loop. Let $P$ denote the Poincaré map $P: l_{0} \rightarrow l_{0}$ which is defined locally on the inside of $S$.

Since there are infinitely many periodic orbits accumulating on $S$ they correspond bijectively to a sequence $u_{i}$ on $l_{0}$ and $\lim u_{i}=0$ as $i \rightarrow \infty$. It is well known [2, p. 293] that

$$
P^{\prime}\left(u_{i}\right)=\exp \int_{0}^{T_{i}} \operatorname{div} X\left(\eta\left(t, u_{i}\right)\right) d t
$$

where $T_{i}$ is the period of $u_{i}$. If $\eta\left(t_{i}, u_{i}\right) \in l_{1}$ (smallest such $t_{i}>0$ ) we have

$$
P^{\prime}\left(u_{i}\right)=\exp \left(\int_{0}^{t_{i}} \operatorname{div} X\left(\eta\left(t, u_{i}\right)\right) d t+\int_{t_{i}}^{T_{i}} \operatorname{div} X\left(\eta\left(t, u_{i}\right)\right) d t\right) .
$$

The second integral is bounded below by $\left(T_{i}-t_{i}\right) \sigma / 2$. The first integral is converging as $i \rightarrow \infty$ to $\int_{0}^{\tau} \operatorname{div} X\left(\eta\left(t, p_{0}\right)\right) d t$. Since $t_{i} \rightarrow \tau$ and $T_{i} \rightarrow \infty$ follows that $P^{\prime}\left(u_{i}\right) \rightarrow$ $+\infty$. In particular, near $S$ all periodic orbits are hyperbolic and unstable, a contradiction. Q.E.D.

The next two theorems begin the classification of the quadratic systems which have a separatrix loop. In particular, if the separatrix loop occurs at a nonhyperbolic critical point $p$ with $\operatorname{div} X(p)=0$, then 4.3 shows that the loop does not surround a limit cycle.

THEOREM 4.2. Let $S$ be a separatrix loop at a critical point $p$ of a quadratic vector field $X$. Then $S$ surrounds a critical point. If $p$ is hyperbolic then $S$ is continuable.

Proof. We may assume $p$ is located at the origin. If $\operatorname{det} d X(p)<0$ then $p$ is hyperbolic, and it easily follows from Theorem 2.4 that $S$ is continuable and consequently surrounds a unique critical point. Hence, suppose $\operatorname{det} d X(p)=0$. $S$ does not surround a critical point only if int $S$ is an elliptic region for $X$ at $p$. If $d X(p)$ vanishes this is impossible since then $X$ is homogeneous, hence its separatrices lie in straight lines. If $d X(p)$ does not vanish, impossibility follows directly from Lemma 2.5, noting from the proof of the lemma that $X$ does not change direction on the invariant line $l$, hence separatrices of $X$ at $p$ lie either in $l$ or on the opposite side of $l$ from the elliptic sector at $p$. Q.E.D.

THEOREM 4.3. Suppose $S$ is a separatrix loop at a nonhyperbolic critical point $p$ such that $\operatorname{div} X(p)=0$, and let $q$ denote the unique critical point in int $S$. Then, there is an invariant line through $p$. Moreover, (a) if $\operatorname{div} X(q)=0$, then $q$ is a center and the orbit through every point in int $S$ is closed; but, (b) if $\operatorname{div} X(q) \neq 0$, then $q$ is a hyperbolic focus and the orbit through every point (except $q$ ) in int $S$ runs from $q$ to $p$ in forward (or reverse) time.

Proof. If $p$ is nonhyperbolic then there is a separatrix loop at $p$ only if $\operatorname{det} d X(p)=0$. Suppose $\operatorname{div} X(p)=0$ as well. We may assume $p$ is at the origin, 
and (by a linear change of coordinates if necessary) that $X$ has the form

$$
X(x, y)=\left[y+a x^{2}+b x y+c y^{2}\right] \partial / \partial x+\left[d x^{2}+e x y+f y^{2}\right] \partial / \partial y .
$$

We claim that $d=0$. For if $d \neq 0$ then the curve along which $X$ is vertical is $y=\phi(x)=0 \cdot x-a x^{2}+\cdots$, and the vertical component along $y=\phi(x)$ is $\psi(x)$ $=d x^{2}+\cdots$. Theorem 67 of Andronov et al. [1, p. 362] implies that the phase portrait of $X$ near $p$ is the union of two hyperbolic sectors and two separatrices, and that both separatrices are tangent to the same ray along the $x$-axis with initial point $p$. But then the fact that $X$ points to the same side of the $x$-axis along its entire length implies that there is no loop at $p$. Thus $d=0$, the $x$-axis is invariant and $X(x, 0)$ points in the same direction for all $x \neq 0$. Moreover, there is only one critical point $q$ of $X$ other than the one at $p$. By Theorem 4.2 there is one. By a linear change of coordinates as in the proof of Theorem 3.2 we may move the invariant line to $l$ : $x+1=0$, move $p$ to $(-1,0)$, and move $q$ to $(0,0)$, giving $X$ the form

$$
X(x, y)=(x+1)(r x+s y) \partial / \partial x+\left(b x+c y+b x^{2}+e x y+f y^{2}\right) \partial / \partial y
$$

where $s \neq 0$. But det $d X(-1,0)=\operatorname{tr} d X(-1,0)=0$ forces $r=c-e=0$, hence rescaling by $1 / s$ we have

$$
X(x, y)=(x+1) y \partial / \partial x+\left(m x+n y+m x^{2}+n x y+c y^{2}\right) \partial / \partial y
$$

for some constant $m<0$ (else $(0,0)$ is not a focus or a center) and some new constant $c \neq 0$ (else the line $x+1=0$ is critical).

To finish the proof recall that given a polynomial vector field $X$ central projection of the plane, regarded as $\{(x, y, z) \mid z=1\} \subset \mathbf{R}^{3}$, onto the sphere, regarded as $\left\{(x, y, z) \mid x^{2}+y^{2}+z^{2}=1\right\}$ induces a vector field $\tilde{X}$ on the upper and lower hemispheres which has an analytic extension, also denoted $\tilde{X}$, to the equator of the Poincare sphere. (The analyticity of the extension follows from the fact that $X$ is a polynomial vector field.) The equator is invariant for $\tilde{X}$ and corresponds to the line at infinity for $X$.

Consider the system

$$
Y(x, y)=(x+1) y \partial / \partial x+\left(m x+m x^{2}+c y^{2}\right) \partial / \partial y .
$$

The critical points of $Y$ are $p:(-1,0)$ and $q:(0,0)$, and by the Center Theorem (2.3), $Y$ has a center at $q$. Moreover, the trajectories of $Y$ are symmetric with respect to the $x$-axis (as point sets). We are interested in the trajectories of $Y$ in the half-plane $H=\{(x, y) \mid x+1>0\}$. Applying the classification theorems of Andronov et al. $[1$, Chapter IX] at $p$ we have the following.

(I) When $c<0$ : then $p$ is a topological saddle point. The only critical points of $Y$ on the Poincare equator are a hyperbolic source and sink at the ends of the line $l$ : $x+1=0$, so by symmetry $p$ is on a saddle loop $L$ surrounding $q$. Every nonstationary orbit in int $L$ is closed, and every orbit off $l \cup L \cup$ int $L$ tends from the source at infinity to the sink at infinity.

(II) When $c>0$ : there is an elliptic sector at $p$ in the half-plane $x+1<0$, two parabolic sectors at $p$, and a hyperbolic sector at $p$ in the half-plane $H$. Every trajectory tending to $p$ in either forward or reverse time is tangent to $l$ at $p$. To find 
the separatrices at $p$ we directionally blow-up the critical point at $p$ (i.e., apply either the transformation $x=x^{\prime}, y=x^{\prime} y^{\prime}$ or the transformation $\left.x=x^{\prime} y^{\prime}, y=y^{\prime}\right)$ two times. We find:

(a) When $0<c<\frac{1}{2}$ : the separatrices bounding the hyperbolic sector at $p$ lie in $H$. Since the only critical points on the Poincare equator are hyperbolic saddles at the ends of $l$, by symmetry $p$ lies on a saddle loop $L$ surrounding $q$. Again every nonstationary trajectory in int $L$ is closed, but every trajectory in $H \backslash$ int $L$ tends to $p$ in both forward and reverse time.

(b) When $\frac{1}{2} \leqslant c \leqslant 1$ : the separatrices bounding the hyperbolic sector at $p$ lie in $l$. The only critical points at infinity are hyperbolic $(c<1)$ or topological $(c=1)$ saddles at the ends of $l$, so every nonstationary trajectory in $H$ is closed.

(c) When $c>1$ : again the separatrices bounding the hyperbolic sector at $p$ lie in $l$. Now, however, there are six critical points at infinity: a hyperbolic sink and source at the ends of $l$ and four hyperbolic saddles. The two saddle separatrices that lie in $H$ must by symmetry be the same trajectory, crossing the $x$-axis between $p$ and $q$. Thus there is a number $k,-1<k<0$, such that the orbit through every point on the half-open line segment $\Sigma=\{(x, y) \mid-1<x \leqslant k, y=0\}$ tends to infinity in both forward and reverse time without (again) crossing the $x$-axis, while the orbit through every nonstationary point in $H \backslash o(\Sigma)$ is closed.

By the same computations applied to $X$ we find that the phase portrait of $X$ near $p$ is qualitatively the same as that of $Y$ near $p$ in each case: (I), (II) (a), (b), (c).

If $\operatorname{div} X(q)=n=0$, then $Y=X$ and 4.4(a) follows. Hence, suppose $n \neq 0$. The sine of the angle $\theta(x, y)$ from $Y(x, y)$ to $X(x, y)$ satisfies

$$
\|Y(x, y)\| \cdot\|X(x, y)\| \sin \theta(x, y)=n(x+1)^{2} y^{2}
$$

so that trajectories of $X$ and $Y$ cross at every point (including the $x$-axis) not on the common invariant line $l$. We treat the case $n>0$; the case $n<0$ is similar.

In Case (I) $(c<0)$ no point of the $X$-unstable manifold of $p$ in $H$ can be in $L \cup$ int $L$. For if $r \in L \cup$ int $L$ then $s=\eta(-1, r)$ is on a closed orbit $\gamma$ of $Y$, and (taking into account the fact that the orientation of $\gamma$ is clockwise) $o_{-}(s) \subset$ int $\gamma$ so $o_{-}(r)$ is bounded away from $p$. But if $r \in H \backslash(L \cup$ int $L)$ then $o_{+}(r)$ is bounded away from $p$ by the $Y$-orbit through $r$, so $p$ is not on a separatrix loop of $X$.

Similar arguments show that $p$ is not on a separatrix loop of $X$ in Cases (II)(b) and (II)(c). (Alternatively, directionally blowing up the critical point of $X$ at $p$ twice shows that both separatrices bounding the unique hyperbolic sector at $p$ lie in $l$.)

In Case (II)(a), the same argument as in Case (I) shows that no point in $L \cup$ int $L$ is in the $X$-unstable manifold of $p$. The proof will be completed by showing that the $X$-stable separatrix at $p$ lies in int $L$. To see this, consider the family of lines given by $y=-\varepsilon$, for $\varepsilon>0$, which meet $l$ at $r=(-1,-\varepsilon)$ and which meet $L$, closest to $p$, at $r^{*}=\left(-1+\delta^{*},-\varepsilon\right)$. For each such $\varepsilon$ the vertical component of $X$ on the segment from $r$ to $r^{*}$ computed at $(-1+\delta,-\varepsilon)$ for $0 \leqslant \delta \leqslant \delta^{*}$ is

$$
\begin{aligned}
m(-1+\delta)-n \varepsilon & +m(-1+\delta)^{2}-n(-1+\delta) \varepsilon+c \varepsilon^{2} \\
& =\varepsilon^{2}(c-n(\delta / \varepsilon))+m \delta(\delta-1) .
\end{aligned}
$$


Since the origin is a focus for $X$ it follows that $m<0$ and since $L$ is tangent to $l$ at $p$ it follows that $\lim _{\varepsilon \rightarrow 0} \delta^{*} / \varepsilon=0$. Hence, for $\varepsilon$ sufficiently small the vertical component of $X$ on the segment is positive. But then the curvilinear triangular region $\Delta$ bounded by the line segment in $l$ from $p$ to $r$, by the line segment from $r$ to $r^{*}$ and by the portion of $L$ from $r^{*}$ to $p$ is positively invariant, so all points in $\Delta$ are in the $X$-stable manifold of $p$. That is, all points in $\Delta$ or its boundary lie on orbits of $X$ which are part of a parabolic sector of $p$. The $X$-stable separatrix bounding this parabolic sector and the hyperbolic sector in $H$ must therefore lie in int $L$. Q.E.D.

REMARK 4.4. In the previous proof if $0<c<\frac{1}{2}$ and if $n>0$ is sufficiently small, then $X$ like $Y$ has exactly two critical points at infinity, both hyperbolic saddles. Hence, there must be an $X$-separatrix loop at $p$ and we observe that the behavior in parts (a) and (b) of 4.3 is realized.

We now treat the case that $S$ is a saddle loop containing a single hyperbolic saddle point. As mentioned in the introduction, Dulac validly showed that $S$ is not the accumulation set of infinitely many limit cycles. However, the generality in which he sought to work makes his proof of this fact quite complicated. Thus, because his proof is so difficult to read, as well as for the sake of completeness, we give a revised proof here. First we state and prove a technical lemma.

LEMMA 4.5. If $a(x, y)$ is analytic on a neighborhood $N$ of the square $J=$ $\{(x, y) \mid-1 \leqslant x \leqslant 1,-1 \leqslant y \leqslant 1\}$ then the vector field $X(x, y)=x \partial / \partial x-$ $y[1-y a(x, y)](\partial / \partial y)$ has an integral in $N$ expressible as a series $H(x, y)=$ $\sum_{k=1}^{\infty} y^{k} h_{k}(x) . H$ is continuous in $N$ and differentiable at all points where $x \neq 0$.

Proof. By hypothesis $a(x, y)$ may be expressed as a series $a(x, y)=$ $\sum_{k=0}^{\infty} y^{k} a_{k+1}(x)$, where for each $k, a_{k+1}(x)$ is analytic on a neighborhood of $[-1,1]$. Thus there exist constants $A_{k+1}>0$ such that $\left|a_{k+1}(x)\right|<A_{k+1}$ on a neighborhood of $[-1,1]$ and such that $A(y)=\sum_{k=0}^{\infty} y^{k} A_{k+1}$ converges there. Define a vector field $Y$ by $Y(x, y)=x \partial / \partial x-y[1-y A(y)] \partial / \partial y$. An integral of $Y$ may be obtained by integrating $d x / x=-(1 / y)[1-y A(y)]^{-1} d y$. But $[1-y A(y)]^{-1}=1+y \alpha^{\prime}(y)$ where $\alpha(y)$ is analytic on a neighborhood of $[-1,1]$ and all the coefficients of its power series expansion about 0 are nonnegative. Hence an integral of $Y$ is $F(x, y)=$ $x y e^{\alpha(y)}=x y \sum_{k=0}^{\infty} y^{k} G_{k+1}$ where $G_{1}=1, G_{k}>0$ for $k \geqslant 2$. Define functions $F_{k}(x)$ by $F_{k}(x)=x G_{k}$ for $k \geqslant 1$. Differentiating $F(x, y)=\sum_{k=1}^{\infty} y^{k} F_{k}(x)$ term by term, the fact that it is an integral of $Y$ means the functions $F_{k}(x)$ satisfy

$$
x F_{k}^{\prime}(x)-k F_{k}(x)=-\sum_{j=1}^{k-1}(k-j) A_{j} F_{k-j}(x), \quad k \geqslant 2 .
$$

Define $h_{1}(x)=x$. For $k \geqslant 2$ define $h_{k}(x)$ on $(0, \infty)$ as the unique solution of the differential equation

$$
x h_{k}^{\prime}(x)-k h_{k}(x)=-\sum_{j=1}^{k-1}(k-j) a_{j}(x) h_{k-j}(x)
$$

with initial value $h_{k}(1)=0$. Similarly define $h_{k}(x)$ on $(-\infty, 0)$ with initial value $h_{k}(-1)=0$. Set $h_{k}(0)=0$. Certainly $\left|h_{1}(x)\right| \leqslant\left|F_{1}(x)\right|$ on $[-1,1]$. If this is true for 
$j=1,2, \ldots, k-1$, then on $[-1,0)$

$$
\begin{aligned}
\left|h_{k}(x)\right| & =\left|x^{k}\right|\left|\int_{-1}^{x} t^{-k-1} \sum_{j=1}^{k-1}(k-j) a_{j}(t) h_{k-j}(t) d t\right| \\
& \leqslant\left|x^{k}\right| \int_{-1}^{x}\left|t^{-k-1}\right| \sum_{j=1}^{k-1}(k-j) A_{j}\left|F_{k-j}(t)\right| d t \\
& =x^{k} \int_{-1}^{x} t^{-k-1} \sum_{j=1}^{k-1}(k-j) A_{j} F_{k-j}(t) d t \\
& =-\left[1-(-x)^{k-1}\right] F_{k}(x) \leqslant-F_{k}(x)=\left|F_{k}(x)\right|,
\end{aligned}
$$

and similarly $\left|h_{k}(x)\right| \leqslant\left|F_{k}(x)\right|$ for $x \in(0,1]$. Hence by induction $\left|h_{k}(x)\right| \leqslant$ $\left|F_{k}(x)\right|=|x| G_{k}$ on $[-1,1]$. Using this fact and the analyticity of $F$ we see that the series $\sum_{k=1}^{\infty} y^{k} h_{k}(x)$ converges absolutely and uniformly on $J$, hence defines a function $H(x, y)$ there. It is clear that $\sum_{k=1}^{\infty} \partial\left(y^{k} h_{k}(x)\right) / \partial y$ converges absolutely and uniformly on $J$ as well. The differential equations for $F_{k}$ and $h_{k}$ imply $\left|h_{k}^{\prime}(x)\right|<$ $2 k G_{k}$, so the same result holds for $\sum_{k=1}^{\infty} \partial\left(y^{k} h_{k}(x)\right) / \partial x$ when $x \neq 0$. Thus term-byterm differentiation of $H$ is valid, hence the condition that $H$ be an integral of $X$ may be reduced to $x h_{1}^{\prime}(x)-h_{1}(x)=0$ and to (4). Q.E.D.

THEOREM 4.6 (DulAC). Let $S$ be a separatrix loop at the hyperbolic saddle point $p$ of an analytic vector field $X$. If $S$ is the accumulation set of infinitely many periodic orbits in int $S$, then the orbit through every point in int $S$ sufficiently close to $S$ is closed.

Proof. By Theorem 4.1 we know that infinitely many orbits in int $S$ can accumulate on $S$ only if the eigenvalues of $d X(p)$ have equal moduli, hence we assume that such is the case. Then by a change of scale, if necessary, and an affine change of coordinates we may assume $p=(0,0)$ and

$$
X(x, y)=[x+P(x, y)] \partial / \partial x+[-y+Q(x, y)] \partial / \partial y
$$

where $P$ and $Q$ are analytic functions. Since the stable manifold (resp. unstable manifold) of $X$ at the origin is locally the graph of an analytic function $x=\alpha(y)$ (resp. $y=\beta(x)$ ), the analytic change of coordinates near the origin given by $F(x, y)=(x-\alpha(y), y-\beta(x))$ transforms $X$ to the form

$$
X=x(1+p(x, y)) \partial / \partial x-y(1+q(x, y)) \partial / \partial y
$$

with $p(0,0)=q(0,0)=0$ and both $p$ and $q$ analytic near the origin. Define

$$
r_{1}(x, y)=(1+p(x, y))^{-1}
$$

and

$$
r_{2}(x, y)=r_{1}(x, y)(1+q(x, y)) .
$$

We observe that there is a disk $D$ centered at the origin in which $F, p, q, r_{1}, r_{2}$ are analytic with $r_{2}$ expressible as

$$
r_{2}(x, y)=1+x \gamma^{\prime}(x)+y b_{1}(x)+y^{2} b_{2}(x)+\cdots,
$$


with $\gamma$ and $b_{i}$ analytic and such that $\left(1+x \gamma^{\prime}(x)\right)^{-1}$ is analytic in $D$. We multiply the vector field by $r_{1}$ to obtain

$$
X=x \partial / \partial x-y(1+b(x, y)) \partial / \partial y
$$

with $b(x, y)=x \gamma^{\prime}(x)+y b_{1}(x)+y^{2} b_{2}(x)+\cdots$. Define the analytic homeomorphism $G: D \rightarrow D^{\prime}$ by $G(x, y)=\left(x e^{\gamma(x)}, y\right)=(u, y)$. With this change of coordinates $X$ is expressible in $D^{\prime}$ as

$$
X(u, y)=u\left[1+x \gamma^{\prime}(x)\right] \partial / \partial u-y\left[1+x \gamma^{\prime}(x)+\sum_{k=1}^{\infty} y^{k} b_{k}(x)\right] \partial / \partial y .
$$

Hence by a change of scale, $X$ appears (reverting to $x$ and $y$ for $u$ and $v$ respectively) as

$$
X(x, y)=x \partial / \partial x-y[1-y a(x, y)] \partial / \partial y
$$

where $a(x, y)$ is analytic in $D^{\prime}$. We may assume (by a change of coordinates of the form $u=c x, v=c y$, with $c>0$ sufficiently small) that the function $a(x, y)$ is analytic on a neighborhood of $J=[-1,1] \times[-1,1]$. As in the proof of Lemma 4.5 we may express $a(x, y)$ as a series $a(x, y)=\sum_{k=0}^{\infty} y^{k} a_{k+1}(x)$, where $a_{k+1}(x)$ is analytic on a neighborhood of $[-1,1]$.

Define the functions $\phi_{k}(x)$ by $\phi_{1}(x)=1$, and for $k \geqslant 2, \phi_{k}(x)$ any solution of

$$
x \phi_{k}^{\prime}(x)-(k-1) \phi_{k}(x)+\sum_{j=1}^{k-1}(k-j) a_{j}(x) \phi_{k-j}(x)=0 .
$$

Thus for example $\phi_{2}(x)=-x \int a_{1}(t) / t^{2} d t$. Hence $\phi_{2}(x)$ is analytic in a neighborhood of $x=0$ if the linear term in $a_{1}(x)$ vanishes, but contains a term with $\ln x$ as a factor otherwise. Similarly $\phi_{k}(x), k \geqslant 3$, may not be analytic near 0 depending on certain terms in $a_{1}(x), \ldots, a_{k-2}(x), \phi_{2}(x), \ldots, \phi_{k-2}(x)$. Suppose there exists an integer $m \geqslant 1$ such that $\phi_{1}(x), \ldots, \phi_{m}(x)$ are analytic but $\phi_{m+1}(x)$ is not. (The case $\phi_{k}(x)$ analytic for all $k$ will be treated later.) Then replace $\phi_{m+1}(x)$ by a solution, also termed $\phi_{m+1}(x)$, of

$$
x \phi_{m+1}^{\prime}(x)-m \phi_{m+1}(x)+\sum_{j=1}^{m}(m+1-j) a_{j}(x) \phi_{m+1-j}(x)=C x^{m}
$$

where $C$ is the coefficient of $x^{m}$ in the sum on the left. The new $\phi_{m+1}(x)$ is then analytic, so we may define an analytic function $f(x, y)$ by $f(x, y)=\sum_{k=1}^{m+1} y^{k} \phi_{k}(x)$. It follows that, since $f(x, y)=\left[y+y^{2} \alpha(x, y)\right]$ for some analytic function $\alpha(x, y)$,

$$
\begin{aligned}
& x f_{x}(x, y)-y[1-y a(x, y)] f_{y}(x, y)+f(x, y) \\
& \quad=C x^{m} y^{m+1}+\hat{\eta}(x, y) y^{m+2}=C x^{m}[f(x, y)]^{m+1}+\eta(x, y) y^{m+2}
\end{aligned}
$$

where $\eta(x, y)$ is analytic on a neighborhood of $J$.

Expressing the analytic functions $-y a(x, y)$ and $\eta(x, y)$ as $-y a(x, y)=$ $\sum_{k=0}^{\infty} x^{k} b_{k}(y)$ and $\eta(x, y)=\sum_{k=0}^{\infty} x^{k} c_{k}(y)$, recursively define functions $\psi_{k}(y)$ by

$$
\begin{gathered}
-y\left[1+b_{0}(y)\right] \psi_{0}^{\prime}(y)+\psi_{0}(y)=-y^{m+2} c_{0}(y), \\
-y\left[1+b_{0}(y)\right] \psi_{k}^{\prime}(y)+(k+1) \psi_{k}(y)=-y^{m+2} c_{k}(y)+y \sum_{j=0}^{k-1} b_{k-j}(y) \psi_{j}^{\prime}(y)
\end{gathered}
$$


for $1 \leqslant k<m$, and

$$
\begin{aligned}
-y\left[1+b_{0}(y)\right] \psi_{m}^{\prime}(y)+(m & +1) \psi_{m}(y)=-y^{m+2} c_{m}(y)+y \sum_{j=0}^{m-1} b_{m-j}(y) \psi_{j}^{\prime}(y) \\
& -C\left(y+y^{2} \phi_{2}(0)+\cdots+y^{m+1} \phi_{m+1}(0)\right)^{m+1} \\
& +C\left(y+y^{2} \phi_{2}(0)+\cdots+y^{m+1} \phi_{m+1}(0)+\psi_{0}(y)\right)^{m+1} .
\end{aligned}
$$

It follows directly by induction that that we may choose $\psi_{k}(y)$ so that $\psi_{k}(y)=$ $y^{m+2} d_{k}(y)$, where $d_{k}(y)$ is analytic on a neighborhood of $J, 0 \leqslant k \leqslant m$. Together with the defining equations for $\psi_{k}(y)$, this implies that if we let $g(x, y)$ be the analytic function $g(x, y)=\sum_{k=0}^{m} x^{k} \psi_{k}(y)$, then

$$
\begin{aligned}
x g_{x}(x, y)-y[1-y a(x, y)] g_{y}(x, y)+g(x, y) & \\
= & -y^{m+2}\left(c_{0}(y)+c_{1}(y) x+\cdots+c_{m}(y) x^{m}\right) \\
& +C x^{m}\left[\left(y+y^{2} \phi(0)+\cdots+y^{m+1} \phi_{m+1}(0)+\psi_{0}(y)\right)^{m+1}\right. \\
& \left.-\left(y+y^{2} \phi(0)+\cdots+y^{m+1} \phi_{m+1}(y)\right)^{m+1}\right] \\
& +y^{m+2} x^{m+1} \hat{\mu}(x, y) \\
= & -y^{m+2} \eta(x, y)+C x^{m}\left[(f(x, y)+g(x, y))^{m+1}-(f(x, y))^{m+1}\right] \\
& +y^{m+2} x^{m+1} \mu(x, y)
\end{aligned}
$$

for some function $\mu(x, y)$ which is analytic on a neighborhood of $J$.

Setting $k(x, y)=f(x, y)+g(x, y)$, it follows that $k$ is analytic in a neighborhood of $J$ and satisfies

$$
\begin{aligned}
x k_{x}(x, y)- & y[1-y a(x, y)] k_{y}(x, y)+k(x, y) \\
= & C x^{m} k(x, y)^{m+1}+x^{m+1} y^{m+2} \mu(x, y) .
\end{aligned}
$$

For $|x|$ and $|y|$ sufficiently small the transformation $u=x, v=k(x, y)$ defines an analytic change of coordinates. In the new coordinates

$$
\begin{aligned}
& \dot{u}=\dot{x}=x=u, \\
& \dot{v}=\dot{x} k_{x}(x, y)+\dot{y} k_{y}(x, y)=x k_{x}(x, y)-y[1-y a(x, y)] k_{y}(x, y) \\
&=-k(x, y)+C x^{m} k(x, y)^{m+1}+x^{m+1} y^{m+2} \mu(x, y) \\
&=-v\left[1-C u^{m} v^{m}-u^{m+1} v^{m+1} \hat{\mu}(u, v)\right]
\end{aligned}
$$

since $v=k(x, y)=y+y^{2} \alpha(x, y), \alpha(x, y)$ analytic. If we finally transform by $x=c u, y=c v$, for $c>0$ sufficiently small we have

$$
X(x, y)=x \partial / \partial x-y\left[1-C x^{m} y^{m}-x^{m+1} y^{m+1} \mu(x, y)\right] \partial / \partial y
$$

where $C \neq 0$, and $\mu(x, y)$ is analytic on a neighborhood of $J$.

By Lemma 4.5, $X$ has an integral of the form $H(x, y)=\sum_{k=1}^{\infty} y^{k} h_{k}(x)$. Computing from (4) we have (with obvious modification if $m=1$ ),

$$
\begin{aligned}
h_{1}(x) & =x, \\
h_{s}(x) & \equiv 0, \\
h_{m+1}(x) & =-C x^{m+1} \ln |x|,
\end{aligned}
$$


and, by induction, for $0 \leqslant s \leqslant m-1$,

$$
h_{r m+s}(x)=x^{m+2} \sum_{k=0}^{r} \alpha_{r m+s, k}(x)(\ln |x|)^{k},
$$

where the functions $\alpha_{k, j}(x)$ are analytic on a neighborhood of $[-1,1]$.

We may suppose that $S$ lies in the first quadrant so that its separatrices lie along the portion of the positive semiaxes in $J$. Choose $\varepsilon>0$ so small that the line segments $\Sigma_{1}=\{(x, 1) \mid-\varepsilon<x<\varepsilon\}$ and $\Sigma_{2}=\{(1, y) \mid-\varepsilon<y<\varepsilon\}$ are arcs without contact for $X$. The section map $y=\tau(x)$ from $\Sigma_{1}^{+}=\{(x, 1) \mid 0<x<\varepsilon\}$ to $\Sigma_{2}^{+}=$ $\{(1, y) \mid 0<y<\varepsilon\}$ satisfies

$$
\tau(x)=H(1, \tau(x))=H(x, 1)=\sum_{k=1}^{\infty} h_{k}(x) .
$$

The Poincaré return map $\rho: \Sigma_{1}^{+} \rightarrow \Sigma_{1}$ does not depend on any analytic change of coordinates, hence we may change coordinates back to the original form of the vector field to obtain an analytic section map $\sigma^{\prime}: \Sigma_{2}^{\prime} \rightarrow \Sigma_{1}^{\prime}$, where $\Sigma_{1}^{\prime}$ and $\Sigma_{2}^{\prime}$ are the images of the sections $\Sigma_{1}$ and $\Sigma_{2}$ under the change of coordinates. Returning to the coordinates in which $\tau$ is defined, $\sigma^{\prime}$ will be expressed as some analytic map $\sigma$ : $\Sigma_{2} \rightarrow \Sigma_{1}$. The Poincaré return map $\rho: \Sigma_{1}^{+} \rightarrow \Sigma_{1}$ is $\sigma \circ \tau$.

A point on $\Sigma_{1}^{+}$, with coordinates $(x, 1)$, is on a closed orbit of $X$ in int $S$ if and only if $x$ satisfies $\sigma \circ \tau(x)=x$, hence by (6) if and only if

$$
\sum_{k=1}^{\infty} h_{k}(x)=\sigma^{-1}(x) \text {. }
$$

If we express the analytic function $\sigma^{-1}$ as $\sigma^{-1}(x)=\sum_{k=0}^{\infty} a_{k} x^{k}$, then using the formulas for $h_{k}(x)$ displayed above, condition (7) on the coordinate $x$ is

$$
x-C x^{m+1} \ln x+x^{m+2} \Phi(x)=\sum_{k=0}^{\infty} a_{k} x^{k},
$$

where $\Phi(x)$ is such that $\lim _{x \rightarrow 0} x \Phi(x)$ exists and is finite. Since $\sigma^{-1}(0)=0$ and the coordinate $x$ cannot be zero, we may divide through by $x$ in this last expression, so that a point $p=(x, 1)$ in $\Sigma_{1}^{+}$is on a closed orbit of $X$ in int $S$ if and only if the coordinate $x$ satisfies

$$
1-C x^{m} \ln x+x^{m+1} \Phi(x)=a_{1}+\sum_{k=2}^{\infty} a_{k} x^{k-1} .
$$

Suppose there exists a sequence of points $p_{n}=\left(x_{n}, 1\right)$ on $\Sigma_{1}^{+}$, each on a closed orbit of $X$ in int $S$ and such that $\lim _{n \rightarrow \infty} x_{n}=0$. Since (8) then holds for each $x_{n}$, letting $n$ tend to infinity we conclude that $a_{1}=1$. Hence we may subtract 1 from each side of (8) and divide through by $x$ again. Again the resulting equality holds for each $x_{n}$, hence passing to the limit we have $a_{2}=0$. Similarly $a_{k}=0$ for $3 \leqslant k \leqslant m$ and condition (8) reduces to $-C \ln x+x \Phi(x)=a_{m+1}+\sum_{k=m+2}^{\infty} a_{k} x^{k-m-1}$, which holds for each $x_{n}$. But now passing to the limit we have a contradiction, so it is impossible that there be infinitely many closed orbits in int $S$ accumulating on $S$ if an integer $m$ as described above exists. 
Thus we turn to the case that all the functions $\phi_{k}(x)$ satisfying equation (5) are analytic on a neighborhood of $[-1,1]$. We now define $f(x, y)$ as $f(x, y)=$ $\sum_{k=1}^{\infty} y^{k} \phi_{k}(x)$ and we will show that $f(x, y)$ is analytic. To this end it is advantageous to use complex variables. Since $a(x, y)$ is real analytic on a neighborhood of the square $J, a(x, y)$ is analytic as a function of two complex variables on $|x| \leqslant 1$ and $|y| \leqslant 1$. If $y a(x, y)=\sum_{k=1}^{\infty} y^{k} a_{k}(x), a_{k}(x)=\sum_{n=0}^{\infty} a_{k n} x^{n}$ it follows that $\left|a_{k}(x)\right|$ $\leqslant \sum_{k=0}^{\infty}\left|a_{k n}\right|=A_{k}$ for $|x| \leqslant 1$.

By (5), $f$ is a formal solution of the partial differential equation

$$
x f_{x}-y(1-y a(x, y)) f_{y}+f=0 .
$$

Define $\alpha(y)=\sum_{k=1}^{\infty} y^{k} A_{k}$ and consider the auxiliary equation

$$
x f_{x}-y(1-\alpha(y)) f_{y}+f=0 .
$$

There is an analytic function $\beta(y)$ defined for $|y| \leqslant 1$ such that $y e^{\beta(y)}=\sum_{k=1}^{\infty} y^{k} B_{k}$ is the solution of (10) which is independent of $x$. From equation (5) we compute

$$
\phi_{k+1}(x)=x^{k} \int_{x}^{1} z^{-k+1} \sum_{j=0}^{k-1}(k-j) a_{j+1}(z) \phi_{k-j}(z) d z
$$

integrating along any path in $\{x \in \mathbf{C}|0<| x \mid \leqslant 1\}$.

A straightforward estimate along the path

$$
\gamma(t)=[(1-r) t / 12+r] e^{i(1-t / 12) \theta}
$$

shows

$$
\left|\phi_{k+1}(x)\right| \leqslant \frac{4}{k} \frac{1-r^{k}}{1-r} \sum_{j=0}^{k-1}(k-j) A_{j+1}\left|\phi_{k-j}\right|
$$

for $x=r e^{i \theta}$ and $|x| \leqslant 1$. If $|x| \leqslant \frac{1}{2}$, we obtain

$$
\left|\phi_{k-j}(x)\right| \leqslant \frac{8}{k} \sum_{j=0}^{k-1}(k-j) A_{j+1}\left|\phi_{k-j}(x)\right|
$$

and an easy induction argument using (10) shows

$$
\left|\phi_{k+1}(x)\right| \leqslant 8^{k} B_{k+1} \leqslant 8^{k+1} B_{k+1} .
$$

Since $\phi_{k}(x)=\sum_{n=0}^{\infty} \phi_{k n} x^{n}$, the Cauchy estimates imply $\left|\phi_{k n}\right| \leqslant 8^{k} B_{k}$. The formal power series $f(x, y)=\sum_{n=0}^{\infty} \sum_{k=1}^{\infty} \phi_{k n} y^{k} x^{n}$ satisfies (9). Also

$$
\sum_{n=0}^{\infty} \sum_{k=1}^{\infty}\left|\phi_{k n}\right||y|^{k}|x|^{n} \leqslant \sum_{n=0}^{\infty} \sum_{k=1}^{\infty} 8^{k} B_{k}|y|^{k}|x|^{n}
$$

and the second series converges for $|x|<\frac{1}{8},|y|<\frac{1}{8}$. Hence, the solution $f$ of (9) is real analytic in a neighborhood of the origin.

The transformation $u=x, v=f(x, y)$ defines an analytic change of coordinates near the origin, and by (9) in the new coordinates $X$ is

$$
X(u, v)=u \partial / \partial u-v \partial / \partial v \text {. }
$$

The transformation $x=c u, y=c v$ for $c>0$ sufficiently small makes this expression valid on a neighborhood of $J$. Reverting to the letters $x$ and $y$ for $u$ and $v$ 
respectively, an integral is $H(x, y)=x y$, so that (6) is now $\tau(x)=x$. Thus although $\tau$ is defined on $\Sigma_{1}^{+}$only, i.e. for $x \in(0, \varepsilon)$ it has an analytic extension to $[0, \varepsilon)$. The argument referred to in the introduction now shows that if $\sigma \circ \tau(x)-x=\sigma(x)-x$ $=0$ infinitely often in $\left[0, \frac{\varepsilon}{2}\right], \sigma(x) \equiv x$, hence the orbit through every point in int $S$ sufficiently near $S$ is closed. Q.E.D.

THEOREM 4.7. Let $S$ be a separatrix loop of a quadratic vector field $X$. If $S$ surrounds infinitely many periodic orbits, then $S$ surrounds a center and the orbit through every nonstationary point in int $S$ is periodic.

Proof. If $S$ surrounds infinitely many periodic orbits, then as in the proof of 3.2 we observe that infinitely many of the periodic orbits accumulate on a separatrix cycle, hence on $S$. By $4.1, \operatorname{div} X(p)=0$. If $p$ is not hyperbolic the result follows from 4.3. If $p$ is hyperbolic the result follows from 4.6 and, as in 3.2 , from the fact that the annulus of periodic orbits near $S$ must extend to the critical point in int $S$. Q.E.D.

THEOREM 4.8. If $X$ is a quadratic system and $S$ is a separatrix loop at the critical point $p$, then $S$ is not the accumulation set of infinitely many limit cycles.

Proof. By 2.9 periodic orbits surrounding the critical point in int $S$ must be in int $S$. Hence 4.8 follows from 4.7. Q.E.D.

5. Examples and questions. In this section we provide examples of various quadratic systems realizing the behavior described in the previous sections, and pose several unanswered questions.

EXAMPLE 5.1. A quadratic vector field with a separatrix cycle as described in Theorem 3.1 is

$$
X(x, y)=x(x+3 y-1) \partial / \partial x-y(3 x+y-1) \partial / \partial y .
$$

The three saddles are at $(0,0),(0,1)$, and $(1,0)$ and the center is at $\left(\frac{1}{4}, \frac{1}{4}\right)$.

EXAMPLE 5.2. A quadratic vector field with a separatrix cycle as in Theorem 3.2, part (a) is

$$
X(x, y)=\left(y+x^{2}+y^{2}\right) \partial / \partial x+x y \partial / \partial y .
$$

The only two critical points are $p:(0,0)$ and $q:(0,-1)$. The $x$-axis is invariant, and an application of the procedure sketched in Andronov et al. [1, p. 365] shows that there is an elliptic sector at $p$ in the lower half-plane (and a hyperbolic sector in the upper half-plane). The critical point at $q$ is hyperbolic, and by symmetry of trajectories with respect to the $y$-axis the stable and unstable eigenspaces through $q$, lines $L^{s}$ and $L^{u}$, meet the positive and negative $x$-axis respectively, say at points $s$ and $u$. By Theorem 2.4, the direction of flow across $L^{s}$ at $s$ determines the direction of flow across $L^{s}$ near $q$, so by hyperbolicity of $q$ the stable separatrices of $q$ lie above $L^{s}$. Thus there is a stable separatrix meeting the interior of triangle $q s u$. Similarly an unstable separatrix at $q$ meets the interior of triangle $q s u$, and it easily follows that each separatrix runs between $p$ and $q$. 
EXAMPLE 5.3. A quadratic vector field with a separatrix cycle as in Theorem $3.2(\mathrm{~b})(\mathrm{i})$ is

$$
X(x, y)=(x+1) y \frac{\partial}{\partial x}+\left(-x+\frac{1}{2} y-x^{2}+\frac{5}{2} x y-\frac{1}{2} y^{2}\right) \frac{\partial}{\partial y} .
$$

In the terminology of the proof of the theorem, since $a=m$ and $b-n \neq 0$, it suffices to verify that the parabola $\mathscr{P}$ with equation $x^{2}-2 x y+y^{2}+2 y-1=0$ passes through the point with coordinates $(-1,0)$ and is invariant under the flow induced by $X$. Then the portion of $\mathscr{P}$ in the half-plane $\{(x, y) \mid x>-1\}$ forms a portion of a separatrix cycle joining the saddle node at $(-1,0)$ and the hyperbolic saddle at $(-1,-4)$.

EXAMPLE 5.4. Consider the family of quadratic vector fields

$$
\begin{aligned}
& X_{b}(x, y)=\left(x^{2}+b x y-(1+b) y^{2}\right) \partial / \partial x+\left(y-2 x^{2}+4 x y-3 y^{2}\right) \partial / \partial y, \\
&-1<b<5 .
\end{aligned}
$$

We will show that, for certain values of the parameter $b$ there are members of the family exhibiting a noncontinuable separatrix cycle as in Theorem 3.2(b), part (i), a noncontinuable separatrix loop at a saddle node, and a continuable separatrix loop at a saddle node (a concrete example of this case is $\left[y(x-1)+x^{2}+y^{2}-1\right] \partial / \partial x$ $-x(x-1) \partial / \partial y$, discussed by Andronov et al. [2, pp. 433-436]).

It is simple to verify that for all $b>-1, X_{b}$ satisfies: (1) the point $p:(0,0)$ is a saddle node with stable separatrix $\sigma$ tangent to the $x$-axis and in quadrant two near $p$, and unstable separatrices tangent to the $y$-axis and lying in the left half-plane; let $\rho$ denote the unstable separatrix at $p$ having nonempty intersection with the upper half-plane (see (5) below); (2) the point $q:(1,1)$ is a hyperbolic saddle point with unstable separatrices tangent at $q$ to the line $y=1$ and lying below it (see (6) below), and with stable separatrices tangent at $q$ to the line through $q$ of slope $(3+b) /(2+b)>1$ and lying to its left (compute $X$ at the crossing of this line with the $x$-axis and apply Theorem 2.4); let $\tau$ denote the unstable separatrix at $q$ in the left half-plane formed by this line; (3) the sole remaining critical point is $v$ : $(-(1+b) D, D), D=\left(2 b^{2}+8 b+9\right)^{-1}$, and is a

stable node for $-1<b \leqslant b_{0} \doteq-.8474$,

stable focus for $b_{0}<b<b_{1} \doteq .6861$,

unstable focus for $b_{1} \leqslant b<b_{2} \doteq 4.0975$,

unstable node for $b_{2} \leqslant b \leqslant 5$;

(4) $X$ is horizontal on an ellipse $E$ with horizontal tangents at $p$ and $q$; the flow of $X_{b}$ is out of (respectively, into) int $E$ on the portion of $E$ from $q$ to $v$ (respectively, $v$ to $p)$; (5) for $x \neq 0, X(x, 0)$ points into the lower half-plane; (6) for $x \neq 1, X(x, 1)$ points into the lower half-plane formed by the line $y=1$; (7) for $y \neq 0, X(0, y)$ points into the left half-plane; (8) by (1), (2),(6) and (7), the stable separatrix at $q$ in the half-plane $y<1$ tends to $p$ in reverse time; and (9), there are two critical points at infinity, a hyperbolic source and a hyperbolic sink, located at the ends of a line with positive slope greater than 1 .

For every number $0<c<1$ let $l_{c}$ denote the portion of the line $y=c$ to the left of $E$; it is an arc without contact for $X_{b}$, all $b>-1$. When they exist let $S_{c}=S_{c}(b)$ 
denote the abscissa of the first intersection in reverse time of $\sigma$ with $l_{c}$, and $T_{c}=T_{c}(b)$ the abscissa of the first intersection of $\tau$ with $l_{c}$. Given $b$, clearly $S_{c}(b)$ exists for $c<D(b)+\varepsilon_{1}(b)$, some $\varepsilon_{1}>0$ and $T_{c}(b)$ exists for $c>D(b)-\varepsilon_{2}(b)$, some $\varepsilon_{2}>0$.

The angle $\theta(x, y)$ from $X_{b}(x, y)$ to $X_{b^{\prime}}(x, y)$ satisfies

$$
\left\|X_{b}(x, y)\right\| \cdot\left\|X_{b^{\prime}}(x, y)\right\| \sin \theta(x, y)=\left[y-2 x^{2}+4 x y-3 y^{2}\right](x-y)\left(b-b^{\prime}\right) y \text {. }
$$

Thus as $b$ monotonically increases, $\theta(x, y)$ is negative throughout a region containing the portions of $\sigma$ and $\tau$ from $p$ and $q$ to $l_{c}$ respectively, so that $S_{c}(b)$ monotonically increases and $T_{c}(b)$ monotonically decreases, each varying continuously with $b$. It follows that if $S_{c}(b)$ exists, so does $S_{c}\left(b^{\prime}\right)$ for $-1<b^{\prime} \leqslant b$, while if $T_{c}(b)$ exists, so does $T_{c}\left(b^{\prime}\right)$ for $b \leqslant b^{\prime} \leqslant 5$.

When $b=b_{0}, \sigma$ must tend to the source at infinity in reverse time, else it tends to $p$, forming a separatrix loop at $p$ surrounding the node at $v$, which is impossible (see the remark at the end of $\S 2)$. Then $\tau$ must tend to $v$, so $S_{1 / 2}(b)<T_{1 / 2}\left(b_{0}\right)$, hence by continuity $S_{1 / 2}(b)$ and $T_{1 / 2}(b)$ exist and

$$
S_{1 / 2}(b)<T_{1 / 2}(b) \text { for } b_{0} \leqslant b<b_{0}+\varepsilon
$$

for some $\varepsilon>0$.

At $b=5, v:(-6 / 99,1 / 99)$ is an unstable node, hence it is not surrounded by a closed orbit, so $\tau$ must tend to the sink at infinity, and $\sigma$ (in reverse time) to $v$. Thus $T_{.01}(5)<S_{.01}(5), T_{.01}(b)$ and $S_{.01}(b)$ exist and

$$
T_{.01}(b)<S_{.01}(b) \text { for } b_{4}-\delta<b \leqslant 5
$$

for some $\varepsilon>0$.

Clearly we may choose $.01=c_{0}<c_{1}<\cdots<c_{K}=\frac{1}{2}$ and intervals $\left[m_{0}, n_{0}\right]$, $\ldots,\left[m_{k}, n_{k}\right]$ such that $t_{c_{j}}$ and $S_{c_{j}}$ exist on $\left[m_{j}, n_{j}\right], 0 \leqslant j \leqslant K, m_{j}<n_{j+1}, 0 \leqslant j \leqslant K$ $-1, n_{0}=5$, and $m_{K}=b_{0} . T_{c}(b)<S_{c}(b)$ implies $T_{c^{\prime}}(b)<S_{c^{\prime}}(b)$ for every $c^{\prime}$ at which the latter values exist (as seen by applying the Jordon Curve theorem to portions of $\sigma$ and $\tau$ from $p$ and $q$ to $y=c$ and $y=c^{\prime}$ together with the saddle separatrix between $p$ and $q$ ), and similarly with inequalities reversed. Hence by $\left(\mathrm{E}_{1}\right)$ and $\left(\mathrm{E}_{2}\right)$ and construction of the $c_{j}$ and $\left[m_{j}, n_{j}\right]$ there exists a $c_{k}$ such that $S_{c_{k}}\left(m_{j}\right)<T_{c_{k}}\left(m_{j}\right)$ and $T_{c_{k}}\left(n_{k}\right)<S_{c_{k}}\left(n_{k}\right)$, hence by continuity $S_{c_{k}}\left(b^{*}\right)=T_{c_{k}}\left(b^{*}\right)$ for some $b^{*} \in\left(m_{k}, n_{k}\right)$. This $X_{b^{*}}$ has a noncontinuable separatrix cycle as described in Theorem 3.2(b), part (i). If $R_{c}(b)$ similarly denotes the abscissa of the first intersection of $\rho$ with $l_{c}$, it is clear that $R_{D\left(b^{*}\right)}\left(b^{*}\right)$ exists and $S_{D\left(b^{*}\right)}<R_{D\left(b^{*}\right)}\left(b^{*}\right)$, so that for $\tilde{b}=b^{*}+\gamma, \gamma>0$ sufficiently small, $\sigma$ tends to $p$ in reverse time, and $X_{\tilde{b}}$ exhibits a noncontinuable separatrix loop at the saddle node $p$.

Finally, let $s(b)$ and $r(b)$ denote the points of first intersection of $\sigma$ and $\rho$ respectively with the portion of $E$ from $v$ to $q$, which exist and depend continuously on $b$ for $b^{*}<b \leqslant b_{2}$ (taking $s\left(b_{2}\right)=v$ ). Then $r(\tilde{b})$ is closer to $v$ (measured along $E$ ) than $s(\tilde{b})$. But at $b_{2}, v$ is an unstable node, hence $\rho$ tends to the sink at infinity, so $s\left(b_{2}\right)$ is closer to $v$ than $r\left(b_{2}\right)$. Consequently there exists $b^{* *}, b^{*}<b^{* *}<b_{2}$, at which $s\left(b^{* *}\right)=r\left(b^{* *}\right)$. This $X_{b^{* *}}$ exhibits a continuable separatrix loop at the saddle node $p$. 
ReMARK. At $b=b_{1}, v$ is a multiple focus; the third focal value is positive, hence a unique unstable limit cycle collapses into $v$ as $b$ increases across $b_{1}$. Since $\operatorname{div} X(0,0)$ $=1>0$, the separatrix loop of $X_{b^{* *}}$ is unstable and as $b$ increases across $b^{* *}$ a unique unstable limit cycle is created from the separatrix loop, onto which $\sigma$ winds in reverse time. Thus it is likely (and numerical integration indicates) that $b^{* *}<b_{1}$, so that we cannot conclude existence of a separatrix cycle surrounding a limit cycle from this example.

EXAMPLE 5.5. A quadratic vector field with a separatrix cycle as described in Theorem 3.2, part (b)(ii) and surrounding a center is any Hamiltonian system which has as its Hamiltonian a cubic polynomial, one level set of which includes a straight line and a parabola crossing at two points. A specific example is the vector field

$$
X(x, y)=2(x+1) y \partial / \partial x-\left(2 x+y^{2}\right) \partial / \partial y
$$

which has as its Hamiltonian $H(x, y)=(1+x)\left(1-x-y^{2}\right)$. The saddles are at $p$ : $(-1, \sqrt{2})$ and $q:(-1,-\sqrt{2})$, and there is a center at $v:(0,0)$.

EXAMPLE 5.6. A quadratic vector field with a separatrix cycle as described in Theorem 3.2, part (b)(ii) and surrounding a focus is more difficult to obtain. We will show that there is a value of the parameter $n$ in the interval $\left(-\frac{2}{3}, 1\right)$ for which the vector field

$$
X(x, y)=(x+1) y \partial / \partial x+\left(-x+n y-\frac{2}{3} x^{2}+x y-\frac{1}{2} y^{2}\right) \partial / \partial y
$$

gives the desired behavior.

For $n \in\left(-\frac{2}{3}, 1\right),(0,0)$ is a focus (when $n=0$, the Center Theorem shows $(0,0)$ is not a center) and the line $l: x+1=0$ is invariant. There are hyperbolic saddles at $p$ :

$$
\left(-1,(n-1)+\sqrt{(n-1)^{2}+2 / 3}\right)
$$

in the upper half-plane and at $q$ :

$$
\left(-1,(n-1)-\sqrt{(n-1)^{2}+2 / 3}\right)
$$

in the lower half-plane (Berlinskiư's Theorem (2.2)). The stable manifold of $p$ lies in the invariant line $l$; denote that portion of its unstable manifold in the half-plane $x>-1$ by $\sigma$. The unstable manifold of $q$ also lies in $l$; denote the portion of its stable manifold lying in the half-plane $x>-1$ by $\tau$. Let $s(n)$ (respectively $t(n)$ ) denote the abscissa of the point of intersection of $\sigma(\operatorname{resp} . \tau)$ with the positive $x$-axis. Let $\tilde{X}$ be the extension of $X$ of the Poincare sphere. For every value of $n, \tilde{X}$ has a single antipodal pair of critical points on the equator, a source at $(0,1,0)$ and a sink at $(0,-1,0)$. Thus $X$ has a single source (in the direction of the positive $y$-axis) and sink (in the direction of the negative $y$-axis) at infinity, for all $n$.

Now let

$$
Y(x, y)=(x+1) y \frac{\partial}{\partial x}+\left(-x-\frac{2}{3} x^{2}-\frac{1}{2} y^{2}\right) \frac{\partial}{\partial y}=-H_{y}(x, y) \frac{\partial}{\partial x}+H_{x}(x, y) \frac{\partial}{\partial y}
$$


for the Hamiltonian

$$
\begin{aligned}
H(x, y) & =-\frac{2}{9} x^{3}-\frac{1}{2} x y^{2}-\frac{1}{2} x^{2}-\frac{1}{2} y^{2}+\frac{5}{18} \\
& =(x+1)\left(-\frac{2}{9} x^{2}-\frac{1}{2} y^{2}-\frac{5}{18} x+\frac{5}{18}\right),
\end{aligned}
$$

whose zero level set is the line $l: x+1=0$ and the ellipse $E$ :

$$
4 x^{2}+9 y^{2}+5 x-5=0,
$$

which intersect at two points. Thus the portion of $E$ in the half-plane $x+1 \geqslant 0$ and the portion of $l$ in int $E$ form a separatrix cycle for $Y$ surrounding a center at $(0,0)$. Call the interior of this separatrix cycle $R$. Note that $E$ intersects the positive $x$-axis at $x=\frac{1}{8}[\sqrt{105}-5]$.

The sine of the angle $\theta(x, y)$ from $Y(x, y)$ to $X(x, y)$ satisfies

$$
\|X(x, y)\| \cdot\|Y(x, y)\| \sin \theta(x, y)=y^{2}(x+1)(x+n),
$$

so that nonstationary trajectories of $X$ and $Y$ cross at every point of the plane not on the vertical lines $x=-1$ and $x=-n$. This implies that $X$ has no closed orbits in the half-plane $x>-1$ when $n=-\frac{2}{3}<-\frac{1}{8}[\sqrt{105}-5]$ and when $n=1$, at which values the line $x=-n$ does not intersect $R$. This is because any closed orbit of $X$ in the half-plane $x>-1$ would have to surround $(0,0)$, hence cross the $x$-axis at a point with abscissa between -1 and 0 , hence cross some closed orbit of $Y$, which is impossible. Thus at $n=-\frac{2}{3}$, since $(0,0)$ is a hyperbolic stable focus, either $\sigma=\tau$ or in reverse time $\tau$ tends to the source at infinity; hence $s\left(-\frac{2}{3}\right) \leqslant t\left(-\frac{2}{3}\right)$. At $n=1$, $(0,0)$ is a hyperbolic unstable focus, and either $\tau=\sigma$ or in forward time $\sigma$ tends to the sink at infinity; hence $S(1) \geqslant t(1)$.

Let $n$ increase from $-\frac{2}{3}$ to 1 . For $n_{1}<n_{2}$ the sine of the angle from $X_{n_{1}}(x, y)$ to $X_{n_{2}}(x, y)$ is positive for $(x, y)$ in the half-plane $x>-1$, so that as $n$ increases $X(x, y)$ turns counterclockwise at every $(x, y)$ with $y \neq 0$ and $x>-1$. It is well known that $s(n)$ and $t(n)$ depend continuously on $n$, and that since the positive $x$-axis is an arc without contact for $X$ and $X$ is rotating counterclockwise, $t(n)$ is strictly decreasing while $s(n)$ is strictly increasing. Thus there is a unique $n_{0} \in\left(-\frac{2}{3}, 1\right)$ at which $s\left(n_{0}\right)=t\left(n_{0}\right)$. This value of $n$ clearly provides the example sought.

We remark that the stability of the separatrix cycle of $X$ is determined by the eigenvalues of $d X$ at $p$ and $q$, and for all $n<1$ is stable on the inside. Thus the separatrix cycle is stable at $n_{0}$. If one could determine that $n_{0}<0$, then one would have established the existence of a limit cycle surrounded by a separatrix cycle. However, we have numerically integrated $X$ and our computations indicate that $n_{0}>0$.

EXAmple 5.7. As usual it is simple to find an example of a saddle loop surrounding a center. A quadratic vector field with a saddle loop surrounding a focus is provided in Andronov et al. [2, p. 427]. By an argument like that of Example 5.6 they show that for a unique number $\mu=\mu_{0} \in(-1,3)$ the vector field

$$
X(x, y)=y \partial / \partial x+\left(-x+\mu y+x^{2}+x y+y^{2}\right) \partial / \partial y
$$


has a saddle loop at the critical point $(1,0)$ which surrounds a focus at $(0,0)$. In fact, the saddle loop is unstable on the inside, so that if $\mu_{0}$ were nonnegative, $(0,0)$ would also be unstable and the existence of a limit cycle inside the loop would be forced. However, by a careful computation of the characteristic exponent of any closed orbit, using the fact that $y=\dot{x}$ as in the proof of 3.2, we can show that if $\mu \geqslant 0$ then every closed orbit is (hyperbolic) unstable, which is impossible. Thus $\mu_{0}<0$ and the question below remains open.

Question. Is there an example of a quadratic vector field having a limit cycle surrounded by a separatrix cycle?

Theorem 3.1 and the examples suggest:

Conjecture. The answer is "no".

A polynomial vector field can have a limit cycle surrounded by a continuable separatrix cycle. The following example was shown to us by David Hart.

EXAMPLE 5.8. The quadratic system

$$
X(x, y)=y \partial / \partial x-\left(x^{2}+x\right) \partial / \partial y
$$

has Hamiltonian $H(x, y)=\frac{1}{3} x^{3}+\frac{1}{2} x^{2}+\frac{1}{2} y^{2}$ and corresponds to the second-order equation

$$
\ddot{x}+x^{2}+x=0 .
$$

$X$ has a hyperbolic saddle at $(-1,0)$ with a saddle loop surrounding a center at $(0,0)$. The equation of the loop is $H(x, y)=\frac{1}{6}$ and $H(x, y)=\frac{1}{2}$ is the equation of one of the periodic orbits inside. Add a dissipative term to (11) to form

$$
\ddot{x}+\dot{x}\left(H(x, y)-\frac{1}{6}\right)\left(H(x, y)-\frac{1}{12}\right)+x^{2}+x=0
$$

with corresponding vector field

$$
Y(x, y)=y \partial / \partial x-\left[\left(H(x, y)-\frac{1}{6}\right)\left(H(x, y)-\frac{1}{12}\right) y+x+x^{2}\right] \partial / \partial y .
$$

Then, $Y$ has a limit cycle corresponding to the curve $H(x, y)=\frac{1}{12}$ surrounded by the separatrix cycle corresponding to the curve $H(x, y)=\frac{1}{6}$. Of course, $Y$ is a polynomial vector field of degree seven.

Finally, a polynomial vector field might have infinitely many limit cycles accumulating at infinity. Here, as in the case with separatrix cycles, arguments previous to those given here concentrate on the analyticity of the system.

In his paper Dulac [6] refers to polynomial (rather than merely analytic) vector fields only because they have finitely many critical points and because they always extend analytically to vector fields on the Poincaré sphere. Unfortunately, a quadratic vector field which has a separatrix cycle containing an orbit at infinity does not generally admit a change of coordinates which places the whole separatrix cycle into the finite plane and which leaves the resulting vector fields a polynomial. This fact prevents the immediate extension of our results to such separatrix cycles. These considerations also point out a fundamental difficulty in Hilbert's problem: where does one use the fact that the vector field is a polynomial and not just an analytic vector field? 
ADDED IN PROOF. After this paper was written Rodrigo Bamón proved the existence of a quadratic vector field with a limit cycle surrounded by a separatrix loop at a hyperbolic saddle point. Thus, the conjecture in $\$ 5$ is false.

\section{REFERENCES}

1. A. Andronov et al., Qualitative theory of second order dynamic systems, Wiley, New York, 1973.

2. __ Theory of bifurcations of dynamic systems on the plane, Wiley, New York, 1973.

3. C. Chicone and J. Tian, On general properties of quadratic systems, Amer. Math. Monthly 89 (1982), $167-178$.

4. W. Coppel, A survey of quadratic systems, J. Differential Equations 2 (1966), 293-304.

5. L. Cherkas, The absence of limit cycles for differential equation with a stable focus, Differential Equations 6 (1970), 779-783.

6. H. Dulac, Sur les cycles limites, Bull. Soc. Math. France 51 (1923), 45-188.

7. P. Hartman, Ordinary differential equations, Wiley, New York, 1964.

8. D. Hilbert, Mathematical problems, Bull. Amer. Math. Soc. 8 (1902), 437-479.

9. S. Lefschetz, Differential equations: Geometric theory, 2nd ed., Interscience, New York, 1963.

10. H. Poincaré, Mémoire sur les courbes définies par une equation différentielle, J. Mathématiques 7 (1881), 375-422.

11. J. Reyn, A stability criterion for separatrix polygons in the phase plane, Nieuw Arch. Wisk. (3) 27 (1979), 238-254.

Department of Mathematics, University of Missouri, Columbia, Missouri 65211

Department of Mathematics, University of North Carolina, Charlotte, North Carolina 28223 